\title{
Analysis of a Three-dimensional Point Pattern with Replication
}

\author{
By A. J. BADDELEY†, \\ Centre for Mathematics and Computer Science, Amsterdam, The Netherlands
}

R. A. MOYEED,

University of Lancaster, UK

C. V. HOWARD

University of Liverpool, UK

and A. BOYDE

University College London, UK

[Received August 1991. Revised August 1992]

\begin{abstract}
SUMMARY
Techniques for analysing three-dimensional spatial point patterns are demonstrated on data from a confocal microscope recording the locations of cells in three dimensions. New computational techniques are proposed for edge corrections and empty space measurement. A novel feature of the data is replication and nesting in a sampling design: multiple spatial patterns were observed from each of several animals. For this we develop a ratio regression approach.
\end{abstract}

Keywords: Confocal microscopy; Distance transform; Empty space function; $K$-function; Monte Carlo inference; Osteocyte lacunae; Replicated spatial patterns; Spatial statistics; Tandem scanning reflected light microscope; Three-dimensional imaging

\section{Introduction}

The three-dimensional structure of living tissue and solid materials can now be observed directly by imaging techniques such as confocal microscopy (Wilson, 1990). Existing statistical methods for analysing 'spatial' point patterns (Ripley $(1981,1988)$, Diggle (1983) and Cressie (1991), chapter 8) are mostly two dimensional; although the general theory works in $d$ dimensions, there are problems for $d>2$ with the complexity of edge correction, increased bias and variance due to edge effects, and extra computational load.

At the same time, the increased ability to capture and store data and to navigate through the three-dimensional material has made it possible to collect replicated samples of a spatial pattern, e.g. three-dimensional images of several different locations in the material. Replication was not studied in the earlier statistical theory; indeed its absence caused difficulties.

$\dagger$ Address for correspondence: Centre for Mathematics and Computer Science, PO Box 4079, 1009 AB Amsterdam, The Netherlands. 
This paper is a worked example of the analysis of a three-dimensional spatial pattern with replication. Our data are three-dimensional co-ordinates giving the positions of osteocyte lacunae in the skull bones of Macaque monkeys observed using a tandem scanning reflected light microscope at University College London (Howard et al., 1985; Baddeley et al., 1987). Osteocyte lacunae are holes in solid bone, occupied by bone cells in life. Several sampling volumes (called 'bricks') were taken within each animal skull, sufficiently far apart to assume independence, and the positions of the lacunae within each brick were recorded. The main scientific questions concerned

(a) the density of lacunae per unit volume, and whether this density is uniform,

(b) spatial patterns in the arrangement of neighbouring lacunae and

(c) variability in lacunar density and spatial pattern within and between animals.

The three-dimensional version of the standard $K$-statistic was described in Baddeley et al. (1987). Braendgaard and Gundersen (1986), Bjaalie and Diggle (1990) and Diggle et al. (1991) have analysed two-dimensional projections of threedimensional patterns, obtained from physical sections. Recently König et al. (1991) have described three-dimensional versions of the $F$-, $G$ - and $K$-statistics. In the present paper we focus on implementation and statistical properties of these estimators.

Replicated data from a confocal microscope were first collected by Howard et al. (1985) and a ratio regression approach was developed in Baddeley et al. (1987). König et al. (1991) found huge variations in estimates of point density $\lambda$ between replicated three-dimensional samples and concluded that their data (three replicates per animal) were insufficient to support quantitative conclusions. Diggle et al. (1991) have developed a bootstrap approach to inference for replicated spatial patterns. Here we shall extend the simpler ratio regression approach to $F$ and $G$ as well as $K$.

A special feature of many three-dimensional spatial data sets is a distinguished direction, the ' $z$-axis', representing for example height or distance from the viewing device. Point patterns may often be regarded as uniform in the other two 'horizontal' directions but not in the $z$-direction, or they may have a different error structure in the $z$-direction. In our data, the $z$-co-ordinate represents depth inside the skull bone and has a definite reference origin (the bone surface) as well as direction; the $x$ - and $y$-coordinates are not so strongly distinguished. This makes the analysis of such data halfway between genuine three-dimensional points and marked two-dimensional points. The standard assumption of stationarity and isotropy may not be valid here.

The data are described in the next section. Section 3 gives some theoretical background and numerical methods. Sections 4 and 5 develop an approach to the replication and nested sampling design. Section 6 records our analysis of the data.

\section{Data}

The experimental technique and sampling protocol are described by Howard et al. (1985) and Baddeley et al. (1987). We examined three intact adult skulls and one calvarium (incomplete skull), all attributed to the Macaque monkey Macaca fascicularis, from the collection of University College London. The focal plane of the confocal microscope was initially positioned $10 \mu \mathrm{m}$ below the cranial surface, then racked down through the bone until no further lacunae could be visualized. The depth $z$ of the centre of each lacuna was determined by adjusting the fine focus racking 
control to yield maximum brightness. The $(x, y, z)$-co-ordinates of the centre of each lacuna were recorded only if $(x, y)$ lay within a graduated rectangular frame fixed to the screen. The three-dimensional sampling volume was therefore a rectangular box, of dimensions $82 \mu \mathrm{m} \times 100 \mu \mathrm{m} \times d \mu \mathrm{m}$, called a brick. The depth $d$ varied from brick to brick.

10 bricks per animal were examined, arranged approximately in a rectangular grid pattern, with at least one brick width separating each pair of bricks. The initial brick position was determined randomly by applying a randomly generated co-ordinate shift to the moving stage. Subsequent bricks were reached by using the coarse controls of the microscope stage, in accordance with the grid pattern.

Fig. 1 shows the pattern from brick 10 of animal 4 displayed as a binocular stereo view, in which each point has been displayed as a cube of side $3 \mu \mathrm{m}$. The threedimensional effect can be obtained with a stereoscopic viewer; many individuals can fuse the images without a viewer (see instructions and examples in Marr (1982) and Tufte (1990)). Instructions for preparing stereo pairs are available from the editors of the Journal of Microscopy.

Stereo pairs seem to be accepted as the most faithful presentation of threedimensional data for publication. An alternative is a single perspective view with the points rendered as solid objects with shading cues (using standard packages such as GNUPLOT). For visualization on the computer screen, dynamic graphic techniques such as spinning (MACSPIN, SPLUS) give more insight.

\section{Theory}

General theory of point processes can be consulted in Daley and Vere-Jones (1989); for statistical methods see Ripley (1981, 1988), Diggle (1983), Stoyan et al. (1987), Cox and Isham (1980) and Cressie (1991), chapter 8. In the standard nonparametric approach (Ripley, 1981), simple summary statistics of the pattern are interpreted as unbiased estimates of the corresponding quantities for the point process, under

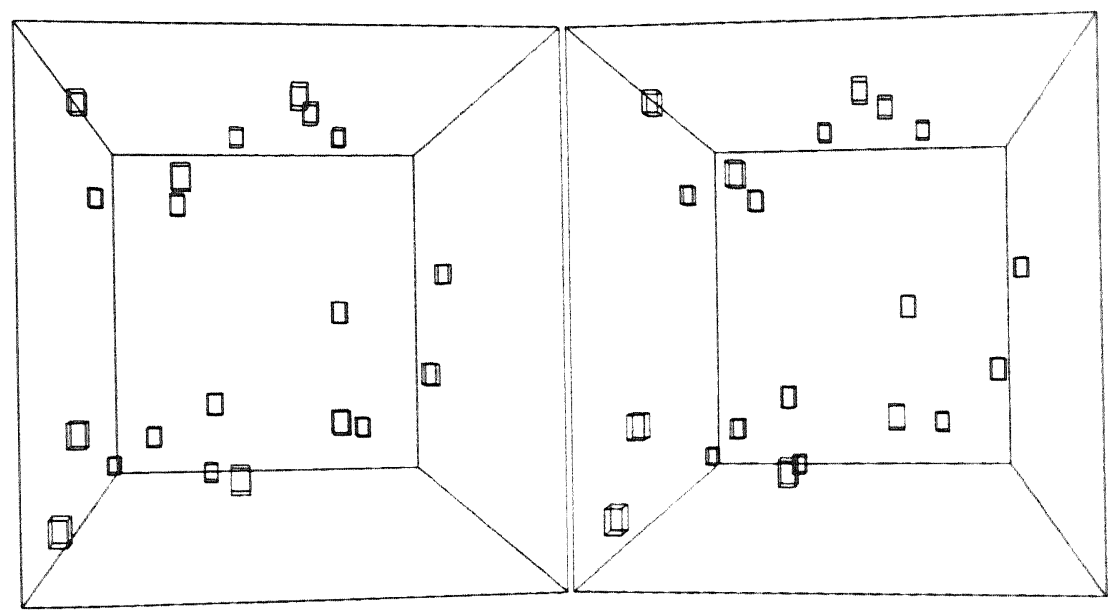

Fig. 1. Stereo pair display of the data in a sampling brick $82 \mu \mathrm{m} \times 100 \mu \mathrm{m}$ wide and $60 \mu \mathrm{m}$ deep, showing each point as a cube of edge $3 \mu \mathrm{m}$ 
minimal assumptions of stationarity. Popular summary statistics for a twodimensional point pattern are the functions $\hat{K}, \hat{G}$ and $\hat{F}$, which are edge-corrected versions of the empirical distributions of (respectively) the distances between all pairs of points in the pattern, the distance from each point in the pattern to its nearest neighbour in the pattern and the distance from a randomly chosen point in the sampling window to the nearest point of the pattern. We shall adapt each of these functions to three dimensions (see also Diggle et al. (1991) and König et al. (1991)).

Estimation is plagued by edge effects resulting from the inability to search outside the sampling window; Miles (1974), Ripley (1988), chapter 3, and Stoyan et al. (1987), section 4.6, give illuminating general discussions. Edge effects are worse in higher dimensions: for example, in a three-dimensional unit cube, the points that are closer than 0.1 units away from the boundary occupy about half the volume. If an unbiased estimate of a parameter is required (e.g. for comparison) there is no alternative but to seek bias corrections, which sometimes involve throwing away data. Corrections for edge effects in $K, G$ and $F$ for two dimensions were given by Ripley $(1981,1988)$ and Hanisch (1984); some of these corrections have been adapted to three dimensions by Baddeley et al. (1987), Diggle et al. (1991) and König et al. (1991). In the present approach, we are further able to exploit the replication to develop efficient unbiased estimators and associated variance estimates.

Edge effects can largely be ignored in hypothesis testing. In the standard Monte Carlo test of a simple null hypothesis, one uses an uncorrected (hence biased) version of a summary statistic, simulates 99 realizations of the null hypothesis and ranks the 100 results according to some one-dimensional criterion. See Ripley (1981), Diggle and Gratton (1984), Hall (1988), Ripley (1988), Diggle et al. (1991) and König et al. (1991).

\subsection{Assumptions}

The point pattern observed inside a sampling region $B$ is taken to be a realization of a spatial point process $\mathbf{N}($ ). For estimations we may want to assume that the process is 'stationary' (invariant under translations of $\mathbf{R}^{3}$ ) and 'isotropic' (invariant under rotations) with regard to moments of order up to $k$ (' $k$ th-order stationarity') or all probability distributions associated with the process ('almost sure stationarity'). Rigorous statements can be found in Stoyan et al. (1987). These are the minimum assumptions in order that the position and orientation of sampling regions need not be recorded, and that replicated data may be pooled. Usually we shall only assume 'horizontal stationarity', i.e. invariance under rotations and translations of the $(x, y)$-plane, and shall test for full invariance.

The usual null hypothesis (and bench-mark for estimation) is that $\mathbf{N}($ ) is a uniform Poisson process with unspecified rate $\lambda>0$ (e.g. Ripley (1981)). The Poisson process serves as the model of complete spatial randomness; departures from Poisson (within the class of almost sure stationary and isotropic processes) are interpreted as indications of 'pattern'. These are traditionally lumped into

(a) 'aggregated' and

(b) 'regular'

alternatives (Diggle, 1983).

For departures from stationarity and isotropy, the natural asymmetry with respect to $z$ suggests a class of alternative hypotheses such as the non-uniform Poisson 
process with rate $\lambda(z)$ depending on $z$ and Gibbs processes with anisotropic interaction potentials (Stoyan et al., 1987).

\subsection{Intensity}

First consider the average density of random points per unit volume. Let $\mathbf{N}(B)$ be the number of random points falling in a region $B \subset \mathbb{R}^{3}$. If $\mathbf{N}()$ is first order stationary then

$$
\mathbf{E N}(B)=\lambda \operatorname{vol}(B)
$$

for a constant $\lambda$ independent of $B$ called the intensity. Thus an unbiased estimator of $\lambda$ is simply the observed density

$$
\hat{\lambda}=\mathrm{N}(B) / \operatorname{vol}(B)
$$

for any window $B$. If $\mathbf{N}()$ is an arbitrary process then

$$
\Lambda(B)=\mathbf{E} \mathbf{N}(B)
$$

defines its intensity measure $\Lambda$. Under mild conditions $\Lambda$ can be represented as the integral of a rate function $\lambda(x, y, z)$. A particularly interesting alternative to stationarity $\lambda(x, y, z)=\lambda>0$ is horizontal stationarity $\lambda(x, y, z)=\lambda_{1}(z) \geqslant 0$. To test the null hypothesis of a stationary Poisson process of unspecified intensity $\lambda$ against the alternative of a horizontally stationary Poisson process with unspecified intensity $\lambda_{1}(z)$, we note that the $z$-co-ordinates of the points in a rectangular box or prism $B$ form a one-dimensional inhomogeneous Poisson process. Conditional on the number of points, the $z$-co-ordinates are independent and identically distributed with probability density proportional to $\lambda_{1}()$, and we can apply standard nonparametric tests for the uniform distribution. More general alternative hypotheses have been discussed by Cressie (1991), p. 657, Lawson (1988) and Ogata and Katsura (1986, 1988).

\subsection{Empty Space Function $F$}

Now consider summary statistics for the spatial pattern on the assumption of stationarity and isotropy. The 'empty space function' $F$ of a point process (almost surely stationary and isotropic) is the probability distribution of the distance from an arbitrary point (say 0 ) to the nearest random point:

$$
F(r)=\mathbf{P}\{\mathbf{N}(S(0, r))>0\}
$$

where $S(x, r)$ is the sphere of radius $r$ around point $x$ in $\mathbf{R}^{3}$ (Ripley, 1981, 1988; Diggle, 1983). $p(r)=1-F(r)$ equals the expected fraction of volume in $\mathbf{R}^{3}$ occupied by points $x$ which are at least $r$ units distant from the nearest random point of the process N( ). For a uniform Poisson process of intensity $\lambda$,

$$
F(r)=1-\exp \left(-\frac{4}{3} \lambda \pi r^{3}\right) \text {. }
$$

Values of $F(r)$ greater than the Poisson value suggest that there is regularity or ordering in the point pattern; lower values suggest aggregation.

Typically $F$ is estimated by taking a fine grid in the sampling region $B$ and computing the distance from each grid point to the nearest observed point of the process. However, edge effects arise because we cannot search for points outside $B$. 
The only unbiased estimator of $F$ that is currently in use is the minus sampling estimator. For a given distance $r$ this considers only points $x$ that are at least $r$ units from the boundary of $B$ and estimates $F(r)$ by the volume fraction of such points which lie closer than $r$ units from some point of the process, i.e.

$$
\begin{aligned}
\hat{F}_{-}(r) & =\frac{\operatorname{vol}\left[B_{(-r)} \cap\left\{\bigcup_{j} S\left(X_{j}, r\right)\right\}\right]}{\operatorname{vol}\left(B_{(-r)}\right)} \\
& =\frac{\operatorname{vol}\left\{x \in B_{(-r)}: \min _{k}\left\|x-X_{k}\right\| \leqslant r\right\}}{\operatorname{vol}\left(B_{(-r)}\right)}
\end{aligned}
$$

where $X_{k}, k=1, \ldots, \mathbf{N}(B)$, are the observed points and

$$
B_{(-r)}=\{x \in B: S(x, r) \subset B\}
$$

is the set of points of $B$ more than $r$ units distant from the boundary of $B$. The points $x$ featuring in the numerator of equation (3) are those for which it is known that the closest random point is within a distance $r$. This estimator is clearly unbiased pointwise for $F$. Minus sampling was first discussed by Miles (1974) for planar problems.

The two-dimensional analogue of equation (3) is typically computed by evaluating, for each point $x$ in a fine rectangular grid, the distance to the nearest point:

$$
d(x)=\min _{k}\left\|x-X_{k}\right\|
$$

and counting the grid points $x$ in $B_{(-r)}$ for which $d(x) \leqslant r$. Diggle and Matérn (1981) discuss the optimal choice of the grid, and Lotwick (1981) describes an algorithm (based on the Dirichlet tessellation) for computing the areas exactly. However, a good approximation to $d(x)$ can be computed very rapidly for all $x$ on a fine rectangular grid by using the distance transform algorithm developed in image processing (Rosenfeld and Pfalz, 1968). This also works in higher dimensions (Borgefors, 1984, 1986). For accurate comparisons the expression (4/3) $\pi r^{3}$ in equation (2) should then be replaced by the discrete volume of the sphere of radius $r$ in this discrete approximation.

Minus sampling throws away part of the data; Diggle (1983) and others prefer to ignore edge effects and use the empirical distribution $\hat{F}_{B}$ of the observed distances $d(x)$. Clearly $\hat{F}_{B}$ is biased for $F$ but can be used in a Monte Carlo significance test of any simple hypothesis. This approach is appealing because it makes full use of the data, but with replicated data we cannot pool $\hat{F}_{B}$ from different bricks $B$ since the bias and variance of $\hat{F}_{B}(r)$ depend on the geometry of $B$. For the Poisson process

$$
\mathbf{E} \hat{F}_{B}(r)=\frac{1}{\operatorname{vol}(B)} \int_{B}(1-\exp [-\lambda \operatorname{vol}\{B \cap S(x, r)\}]) \mathrm{d} x,
$$

whereas if we condition on the number of points $n$ then $1-\exp (-u)$ should be replaced by $1-(1-u)^{n}$. An analytical expression for the integrand is given in Appendix A.

\subsection{Estimation of $G$}

The function $G$ is the distribution of the distance from a typical point of the process to the nearest other point: loosely 


$$
G(r)=\mathbf{P}\{\mathbf{N}(S(x, r))>1 \mid \text { point at } x\}
$$

where $x$ is arbitrary. Under suitable conditions

$$
G(r)=\frac{1}{\lambda \operatorname{vol}(B)} \mathrm{E} \sum_{X_{k} \in B} 1\left\{\mathrm{~N}\left(S\left(X_{k}, r\right)\right)>1\right\}
$$

where $B$ is arbitrary. For a Poisson process $G(r)=1-\exp \left\{-(4 / 3) \lambda \pi r^{3}\right\}$; again, this is taken as a bench-mark for interpretation of empirical $G$-functions. Edge effects arise because we can only observe nearest neighbour distances within $B$,

$$
s_{k}=\min _{X_{i} \in B, l \neq k}\left\|X_{k}-X_{l}\right\|, \quad X_{k} \in B .
$$

Ripley (1981) (see also Ripley (1988), chapter 3) introduced the minus sampling estimator

$$
\hat{G}_{1}(r)=\frac{\sum_{j} 1\left\{s_{j} \leqslant r\right\} 1\left\{X_{j} \in B_{(-r)}\right\}}{\sum_{j} 1\left\{X_{j} \in B_{(-r)}\right\}}
$$

where 1\{\} denotes an indicator variable and $B_{(-r)}$ was defined in equation (4). The denominator is the number of all points lying more than $r$ units from the boundary of $B$, and the numerator is the number of these that have $s_{j} \leqslant r$.

Hanisch (1984) (see also Stoyan et al. (1987), p. 128, and Ripley, (1988), chapter 3) observed that in general $\hat{G}_{1}$ will not be an increasing function and may have values greater than 1. Hanisch developed two further edge-corrected estimators of $G$, of which we shall use

$$
\hat{G}_{3}(r)=\frac{\sum_{j} 1\left\{s_{j} \leqslant r\right\} 1\left\{X_{j} \in B_{\left(-s_{j}\right)}\right\}}{\sum_{j} 1\left\{X_{j} \in B_{\left(-s_{j}\right)}\right\}} .
$$

This consists in restricting both the numerator and the denominator of equation (7) to points $X_{k}$ for which $s_{k}$ is known to be the 'true' nearest neighbour distance, i.e. for which the nearest other point is closer than the boundary. Clearly $\hat{G}_{3}$ is a distribution function; Hanisch (1984) showed that it is pointwise consistent in the limit as the sampling region expands to cover $\mathbf{R}^{3}$.

Edge effects can be severe for $G$, so it becomes appealing to employ the uncorrected empirical distribution $\hat{G}_{B}$ of the observed $s_{k}$. This can be treated in the same way as $\hat{F}_{B}$. For the Poisson process $\mathbf{E} \hat{G}_{B}(r)=\mathbf{E} \hat{F}_{B}(r)$ as given by equation (5), but if we condition on the number $n$ of points then $\exp (-x)$ in equation (5) is replaced by $(1-x)^{n-1}$.

\subsection{Estimation of $K$}

Finally $K(r)$ is the mean number of other points of the process that lie within a radius $r$ of a typical point of the process, divided by the intensity $\lambda$ :

$$
K(r)=\frac{\mathbf{E}\{\mathbf{N}(S(x, r))-1 \mid \text { point at } x\}}{\lambda} .
$$


Under stationarity assumptions

$$
\lambda K(r)=\frac{1}{\lambda \operatorname{vol}(B)} \mathbf{E} \sum_{X_{k} \in B} \sum_{X_{l} \neq X_{k}} 1\left\{\left\|X_{k}-X_{l}\right\| \leqslant r\right\}
$$

(where $X_{l}$ is not restricted to lie inside $B$ ). If the process is also isotropic, $K(r)$ completely determines the second-order moments of the process. For a Poisson process

$$
K(r)=\frac{4}{3} \pi r^{3}
$$

and again this serves as a bench-mark for the interpretation of empirical $K$-functions.

Ripley (1988), pages 31-35 (see also Stoyan et al. (1987), pages 122-126), describes several edge effect corrections for estimating $K$ in two dimensions. Here we consider three-dimensional versions of the 'border', 'translation' and 'isotropic' corrections. The border correction estimate is

$$
\hat{K}_{\mathrm{b}}(r)=\frac{\operatorname{vol}\left(B_{(-r)}\right)}{\mathrm{N}\left(B_{(-r)}\right)^{2}} \sum_{X_{k} \in B_{(-r)}} \sum_{X_{l} \in B} 1\left\{0<\left\|X_{k}-X_{l}\right\| \leqslant r\right\} ;
$$

this is a straight application of minus sampling to equation (9) and is valid for any second-order stationary process. The sum is an unbiased consistent estimator of $\lambda^{2} \operatorname{vol}\left(B_{(-r)}\right) K(r)$ so estimate $(10)$ is consistent and approximately unbiased.

The (Miles-Lantuéjoul-Stoyan-Hanisch) translation-corrected estimate is

$$
\hat{K}_{t}(r)=\frac{\operatorname{vol}(B)^{2}}{\mathrm{~N}(B)^{2}} \sum_{X_{k} \in B} \sum_{X_{l} \neq X_{k}} \frac{1\left\{\left\|X_{k}-X_{l}\right\| \leqslant r\right\}}{\gamma_{B}\left(X_{k}-X_{l}\right)}
$$

where $\gamma_{B}$ is the 'set covariance'

$$
\gamma_{B}(v)=\operatorname{vol}\{x \in B: x+v \in B\}
$$

Again this is valid for any second-order stationary process. If additionally the process is known to be isotropic, then the isotropic correction estimate

$$
\hat{K}_{t}(r)=\frac{\operatorname{vol}(B)}{\mathrm{N}(B)^{2}} \sum_{X_{k} \in B} \sum_{X_{l} \neq X_{k}} \frac{1\left\{\left\|X_{k}-X_{l}\right\| \leqslant r\right\}}{w_{B}\left(X_{k}, X_{l}\right) s_{B}\left(\left\|X_{k}-X_{l}\right\|\right)}
$$

is valid, where $w_{B}\left(X_{k}, X_{l}\right)$ is an edge correction equal to the proportion of the surface area of the sphere with centre at $X_{k}$ and radius $\left\|X_{k}-X_{l}\right\|$ which lies within the sampling window $B$ :

$$
w_{B}(x, y)=\frac{\operatorname{area}\{\partial S(x,\|x-y\|) \cap B\}}{\operatorname{area}\{\partial S(x,\|x-y\|)\}}
$$

where $\partial S(x, r)$ is the surface of the sphere $S(x, r)$. Meanwhile $s_{B}(r)$ is a global geometry correction

$$
s_{B}(r)=\frac{\operatorname{vol}\{x \in B:\|x-y\|=r, \text { some } y \in B\}}{\operatorname{vol}(B)}=\frac{\operatorname{vol}\{x \in B: \partial S(x, r) \cap B \neq \varnothing\}}{\operatorname{vol}(B)} ;
$$

it was first noted by Hanisch (1984) that the term $s_{B}(r)$ is needed for larger $r$. We give analytical expressions for $w_{B}$ and $s_{B}$ in Appendix A for a rectangular box.

The sum in equation (12) is a consistent unbiased estimator of 


$$
Y(r)=\lambda^{2} \operatorname{vol}(B) K(r)
$$

(in the limit as the sampling region expands to cover $\mathbf{R}^{3}$ ); hence estimate (12) is consistent and approximately unbiased.

In two dimensions, Ripley (1981) and Diggle (1983), p. 71, generally recommend that estimates of $K(r)$ should only be employed for $r$ less than half the minimum side length of the sampling rectangle. However, we shall go to larger values of $r$ by pooling information from replicates.

\subsection{Comments}

No one of the functions $F, G$ and $K$ should be regarded as superior to the others, nor as characterizing a point pattern. For example, two quite different processes may have the same $K$-function (Baddeley and Silverman, 1984). This insensitivity is a feature of most current techniques in spatial statistics. Analysis of a point pattern should normally include several measures of interaction.

Alternatives to $F, G$ and $K$ may be proposed if there is a suggestion of anisotropy or directional asymmetry in the pattern. The sphere $S(x, r)$ used in the definitions of $F, G$ and $K$ may be replaced by a cylinder or ellipsoid, so that the statistic becomes a function of two or nine parameters respectively. This is equivalent to replacing the ordinary Euclidean distance $\|a-b\|$ by some other metric. The edge corrections discussed here continue to hold, except for the isotropic correction for $K$. The simplest way to induce an isotropic correction for the ellipsoidal $K$-function is to subject the point pattern data to a linear transformation which maps the ellipse to a sphere, then to apply the isotropic estimator. Note that a linear transformation maps a Poisson process to a Poisson process, but for example a hard core process (Stoyan et al., 1987) is mapped to a process with genuinely anisotropic pattern.

\section{Pooling Replicates by Ratio Estimation}

Our data include replicate observations from 10 bricks in each animal. It may be appropriate to pool across bricks within each animal, and possibly to pool across animals to produce an overall estimate for the animal population. In this section we discuss pooling estimates of $\lambda, K, F$ and $G$ from replicates within each animal, assuming that the bricks are true replicates: in particular, assuming that $\lambda$ is constant across replicates.

It does not seem to have been pointed out in the spatial statistics literature that the usual statistics $\hat{K}, \hat{G}$ and $\hat{F}$ are ratio estimators. Equations (1), (3), (7)-(8) and (10)-(12) define statistics $T$ of the form $T=U / V$ where

$$
t=\mathbf{E} U / \mathbf{E} V
$$

is the parameter of interest. In all but the first two cases, the denominator $V$ is not fixed and $T$ is biased for $t$ : hence the usual remark that $\hat{K}$ and $\hat{G}$ are approximately unbiased and consistent (asymptotically in the size of the sampling region).

It is then appropriate to use ratio regression to estimate $t$ in equation (13). Take the standard additive effects model

$$
U_{j}=t V_{j}+e_{j}, \quad j=1, \ldots, m,
$$

(Cochran, 1977; Cruz Orive, 1980) where the errors $e_{j}$ are conditionally independent given the $V_{j}$, with 


$$
\operatorname{var}\left(e_{j} \mid V_{j}\right) \propto V_{j} \text {. }
$$

The sample mean of the $T_{j}$ is biased for $t$ and is not consistent as the number of replications increases. The ratio of sums

$$
\hat{t}=\sum U_{j} / \sum V_{j}
$$

is unbiased for $t$ and is the best linear unbiased estimator conditional on the $V_{j}$. The bias and variance of equation (16) are to first order (Cochran (1977), pages 161, 154)

$$
\begin{gathered}
\mathbf{E}(\hat{t}-t) \approx \frac{t}{m}\left\{C_{u u}-C_{u v}\right\}, \\
\operatorname{var}(\hat{t}) \approx \frac{t^{2}}{m}\left\{C_{u u}+C_{v v}-2 C_{u v}\right\}
\end{gathered}
$$

where $C_{u u}, C_{u v}$ and $C_{v v}$ are the entries in the covariance matrix of $(U / \mathbf{E} U, V / \mathbf{E} V)$.

The estimator of $\lambda$ in equation (1) is of the form $\hat{\lambda}=U / V$ where $U=\mathbf{N}(B)$ is the number of random points in $B$ and $V=\operatorname{vol}(B)$. In a Poisson point process $\operatorname{var}\{\mathrm{N}(B)\}$ $=\mathbf{E} \mathbf{N}(B)=\lambda \operatorname{vol}(B)$ so expressions (14) and (15) apply. For more general processes, if we can assume that the effective range of spatial dependence is small compared with the size of the sampling region, we can appeal to central limit theorems (e.g. Baddeley (1980) and Jolivet (1980)).

For the estimation of $F(r)$ for fixed $r$ via equation (3), central limit theorems for Poisson superpositions of random sets (see for example Hall (1989)) suggest that the variance of the numerator of equation (3) may be taken as approximately proportional to the denominator. Hence the best estimate of $F(r)$ is the ratio of total numerator to total denominator in equation (3),

$$
\hat{F}_{\text {ratio }}(r)=\frac{\sum_{j=1}^{m} F^{(j)}(r) \operatorname{vol}\left(B_{j(-r)}\right)}{\sum_{j=1}^{m} \operatorname{vol}\left(B_{j(-r)}\right)}
$$

where $F^{(j)}(r)$ is the estimate from data in $B_{j}$, and an estimate of the variance of this estimator may be obtained from approximation (18).

For $K(r)$, take $U$ to be the double sum in each of estimates (10)-(12). Then Ripley (1981, 1988) and Hanisch (see Stoyan et al. (1987)) have shown that $\mathbf{E} U / \mathbf{E} V=K(r)$ for any second-order stationary and isotropic process. Ripley (1979) pointed out that $U_{j}$ is a U-statistic

$$
U_{j}=\sum_{k \neq l} u_{j}\left(X_{j k}, X_{j l}\right)
$$

where the summation is over all ordered pairs of distinct points in pattern $\mathscr{P}_{j}$, and the function $u_{j}$ depends on the geometry of $B_{j}$. Symmetrize $u_{j}$ if necessary. For a uniform Poisson process, since $V_{j}$ is a 1-1 function of $N=\mathbf{N}\left(B_{j}\right)$,

$$
\begin{aligned}
\operatorname{var}\left(U_{j} \mid V_{j}\right) & =\operatorname{var}\left\{U_{j} \mid \mathbf{N}\left(B_{j}\right)\right\} \\
& =2 N(N-1) \operatorname{var}\left\{u_{j}\left(X_{1}, X_{2}\right)\right\}+2 N(N-1)(N-2) \operatorname{var}\left[\mathbf{E}\left\{u_{j}\left(X_{1}, X_{2}\right) \mid X_{1}\right\}\right]
\end{aligned}
$$

where $X_{1}$ and $X_{2}$ are independent uniformly distributed points in $B_{j}$. If edge effects could be ignored and if the $B_{j}$ were all identical, the conditional expectation in the 
second term would be constant and expression (15) would hold. Ripley (1979) showed that in two dimensions the first term indeed dominates, at least for $r$ no greater than a quarter of the smallest edge length of the sampling rectangle. In our case we can assume that expression (15) holds when $r$ is 'sufficiently small' and when the dependence of $u_{j}$ on $B_{j}$ can be neglected. Thus for example in estimate (12) we estimate the pooled $K(r)$ across bricks within one animal by the weighted average of the brick estimates $K_{j}(r)$ weighted by $N\left(B_{j}\right)^{2}$.

Finally, for $G$, the numerator $U$ and denominator $V$ of equation (7) clearly satisfy $\mathbf{E} U / \mathbf{E} V=G(r)$, and Hanisch (1984) showed that the same holds for equation (8). An argument similar to that for $K$ above justifies expression (15), again if we ignore edge effects.

For the count data used in estimating $\lambda, K$ and $G$, we could alternatively take a quasi-likelihood approach assuming $\operatorname{var}(N)=w \mathbf{E} N$, with $w=1$ corresponding to Poisson variation.

\section{Analysis of Variance}

In this section we consider pooling estimates across animals and performing an analysis of variance within animals compared with between animals.

The full experiment analysed here is a one-way nested design yielding point patterns $\mathscr{P}_{i j}$ from bricks $j=1, \ldots, m$ in animals $i=1, \ldots, n$. In place of equation (14) we now have

$$
U_{i j}=t_{i} V_{i j}+e_{i j}
$$

where the $t_{i}$ are independent random effects with mean $t$ and variance $\tau^{2}$, and the $e_{i j}$ are conditionally independent given $t_{i}$, with

$$
\operatorname{var}\left(e_{i j} \mid V_{i j}, t_{i}\right)=w_{i} V_{i j} \text {. }
$$

Here $t$ is the parameter to be estimated, $\tau^{2}$ is the between-animal variance (of the random effects $t_{i}$ ) and $w_{i}$ are nuisance parameters controlling the within-animal variability. The simplification $w_{i}=w$ did not seem justified for our data.

The parameter $t$ is now the population mean of the spatial statistic $\lambda, K(r), F(r)$ or $G(r)$. It is not always clear how to interpret this. The population mean is well defined but, since the values of $F$ and $G$ for a Poisson process depend on $\lambda$, there is no natural bench-mark for the population means of $F(r)$ and $G(r)$ unless $\lambda$ is constant across the population. The $K$-function is adjusted for $\lambda$ and has a natural bench-mark; however, the interpretation of the population mean of $K(r)$ is also affected by variation in $\lambda$.

Our approach is first to estimate $t_{i}$ and $\sigma_{i}^{2}=\operatorname{var}\left(\hat{t}_{i} \mid t_{i}\right)$ by the ratio regression method above, giving values $\hat{t}_{i}$ and $\hat{\sigma}_{i}^{2}$. Then we compute a sequence of estimates $t^{(k)}$, $\tau_{(k)}^{2}$ of $t, \tau^{2}$ for $k=0,1,2, \ldots$ by setting $t^{(0)}, \tau_{(0)}^{2}$ to be the sample mean and variance of the $\hat{t}_{i}$, and iterating

$$
\begin{gathered}
t^{(k+1)}=\frac{\sum \hat{t}_{i} /\left(\tau_{(k)}^{2}+\hat{\sigma}_{i}^{2}\right)}{\sum 1 /\left(\tau_{(k)}^{2}+\hat{\sigma}_{i}^{2}\right)}, \\
\tau_{(k+1)}^{2}=\left(\frac{\sum\left\{\left(\hat{t}_{i}-t^{(k)}\right)^{2}-\sigma_{i}^{2}\right\}\left(\tau_{(k)}^{2}+\hat{\sigma}_{i}^{2}\right)^{-1}}{\sum 1 /\left(\tau_{(k)}^{2}+\hat{\sigma}_{i}^{2}\right)}\right)_{+}
\end{gathered}
$$


where $(x)_{+}=\max \{x, 0\}$. Here equation (22) takes the best linear unbiased estimator of $t$ given observations $\hat{t}_{i}$ with known variances, and equation (23) forms an unbiased estimate of $\tau^{2}$ given $\hat{t}_{i}$ when $t$ and $\sigma_{i}^{2}$ are known. Together equations (22) and (23) are respectively the E-step and M-step of the EM algorithm for $t$ and $\tau^{2}$ in the model where the $\sigma_{i}^{2}$ are known and $\hat{t}_{i}$ are normal, i.e. regarding $s_{i}=t_{i}-t$ as missing observations.

This method will be adopted unless data are scarce, when we shall resort to pooling all data $\left(X_{i j}, Y_{i j}\right)$ and performing a simple ratio regression; this is equivalent to fixing $t_{i}=t, \tau^{2}=0$ and $w_{i}=w$.

Steps (22) and (23) are iterated until convergence to produce estimates $\hat{t}$ and $\hat{\tau}^{2}$. The variance of $\hat{t}$ can be estimated by the sample version of

$$
\operatorname{var}(\hat{t}) \approx 1 / \sum_{i=1}^{n} b_{i}
$$

where $b_{i}=1 /\left(\tau^{2}+\sigma_{i}^{2}\right)$. The contributions to approximation (24) associated with between-animal and within-animal, between-brick variation can be estimated respectively by

$$
\begin{gathered}
v_{\text {between }}=\tau^{2} \sum b_{i}^{2} /\left(\sum b_{i}\right)^{2}, \\
v_{\text {within }}=b_{i}^{2} \sigma_{i}^{2} /\left(\sum b_{i}\right)^{2} .
\end{gathered}
$$

\section{Analysis of Our Data}

\subsection{Intensity}

Fig. 2 is a scatterplot of point count $n_{i j}$ against brick depth $d_{i j}$ (proportional to volume) labelled by animal index $i$. Since brick depth was determined by our ability to see through the material, it might have some association with the spatial pattern and would usually not be ancillary. However, this association was thought neither relevant nor severe, so we condition on the $d_{i j}$ and regard $\mathscr{P}_{i j}$ for $j=1, \ldots, 10$ as independent realizations of the same point process $\mathbf{N}^{(i)}()$ in $\mathbf{R}^{3}$, observed inside predetermined windows $B_{i j}$. The four animals furnish distinct point processes $\mathbf{N}^{(i)}()$.

The ratio regression model (14)-(15) looks plausible across bricks within each animal, but the data for animal 1 appear to have a different slope from the rest.

Table 1 shows the individual estimates $n_{i j} / v_{i j}$ of cell density from each brick, the pooled ratio estimate $\hat{\lambda}_{i}$ for each animal $i$ from equation (16) and the estimated standard deviation $\hat{\sigma}_{i}$ of $\hat{\lambda}_{i}$ from approximation (18).

Applying steps (22) and (23) to the tabulated data gives $\hat{\lambda}=32.3$ with estimated variance 9.1 (standard error 3.0). The within-animal and between-animal variance contributions were 1.1 and 8.0 respectively. The estimate of population betweenanimal variance $\tau^{2}$ was 31.4 (standard deviation 5.6) and a notional within-animal variance per sampling brick is $10 \times 1.1=11=3.3^{2}$.

The data for animal 1 appeared to be self-consistent but in conflict with the (mutually consistent) data for animals 2-4. Given the small number of animals it was difficult to decide whether to regard the result for animal 1 as an outlier. When we repeated the analysis without animal 1, the EM procedure led to a zero estimate for $\tau^{2}$ and weighted mean $\hat{\lambda}=35.1$ with estimated variance 1.5 (standard error 1.2 ).

In the light of this, the bone material was re-examined. Animal 1 was only 


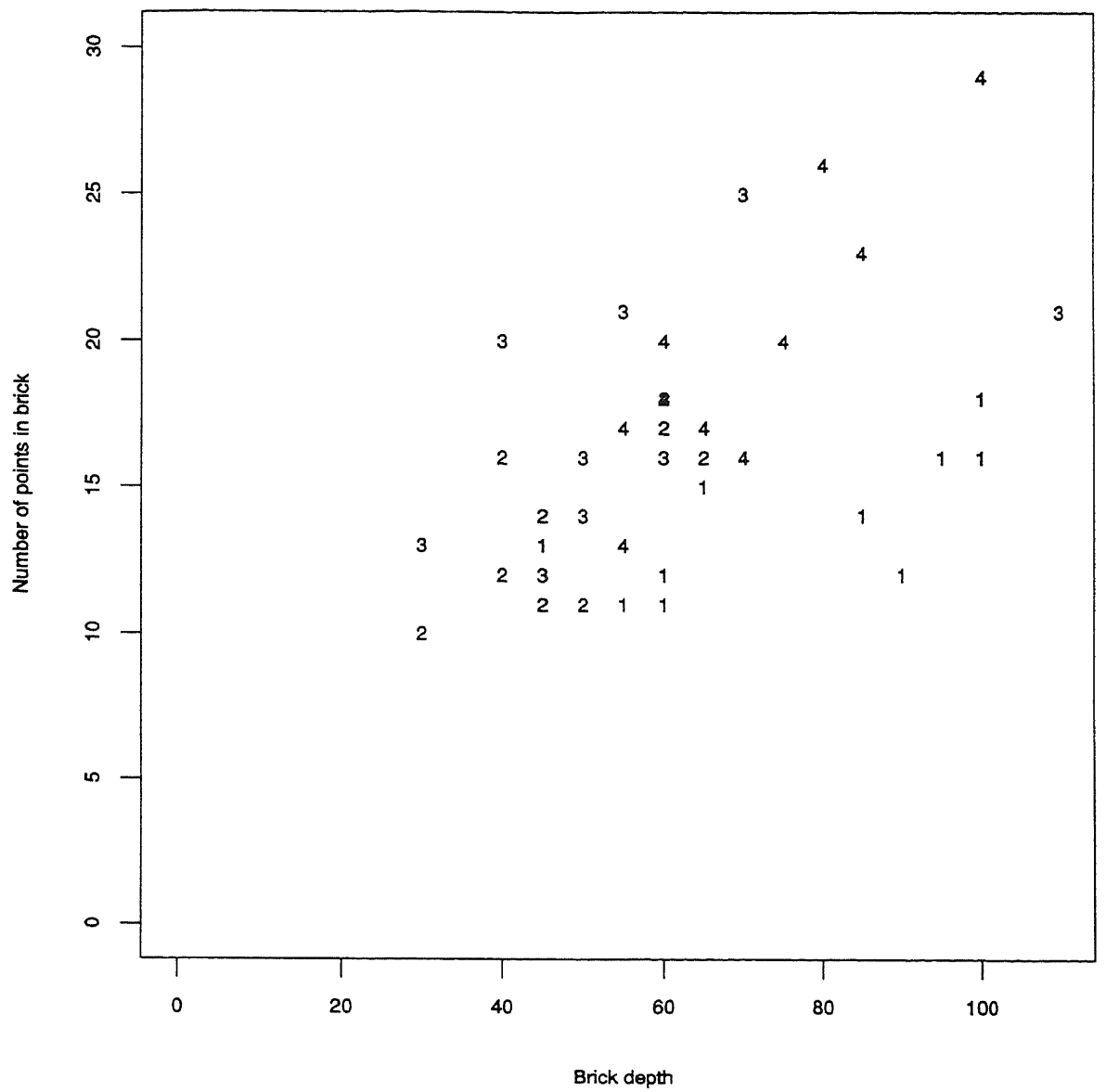

Fig. 2. Scatterplot of cell count $n_{i j}$ against brick depth $d_{i j}$ labelled by animal index $i$

represented by a calvarium (skullcap); from animals 2-4 we had complete intact skulls. Anatomical differences were found on closer examination. The three intact skulls recurved strongly behind the parietal-occipital suture, whereas in animal 1 the skull continued backwards. It was concluded that animal 1 came from a different species, though anatomically not very different. In the subsequent analysis we handle animal 1 separately and pool only across animals $2-4$.

TABLE 1

Estimates of $\lambda$

\begin{tabular}{llllllllllllll}
\hline Animal & & \multicolumn{1}{c}{$\lambda$ estimates $\left(\right.$ per $\left.10^{6}(\mu m)^{3}\right)$} & & & $\hat{\lambda}_{i}$ & $\hat{\sigma}_{i}$ \\
\hline 1 & 35.7 & 22.6 & 24.7 & 24.7 & 20.3 & 16.5 & 20.8 & 28.5 & 22.2 & 19.8 & 22.6 & 1.4 \\
2 & 30.2 & 41.2 & 37.0 & 38.4 & 37.0 & 27.2 & 49.4 & 37.0 & 30.4 & 35.0 & 35.6 & 1.9 \\
3 & 61.7 & 47.1 & 32.9 & 39.5 & 32.9 & 53.5 & 34.6 & 32.9 & 44.1 & 23.6 & 37.8 & 3.9 \\
4 & 37.0 & 32.3 & 29.2 & 28.2 & 38.2 & 35.8 & 40.1 & 32.9 & 33.4 & 41.1 & 34.8 & 1.3 \\
\hline
\end{tabular}




\subsection{Uniformity in z-co-ordinate}

The empirical distribution functions of the depths of the points in each brick were compared with the uniform distribution by using a standard two-sided Kolmogorov-Smirnov test. Of the 40 bricks in the four animals the null hypothesis was rejected at the $5 \%$ level only for the two bricks belonging to animals 2 and 4 . Inspection of the plots showed no strong suggestion of non-uniformity.

\section{3. $K$-function}

Initially the $K$-function was estimated separately from each brick. Fig. 3 shows a comparison of $K$-estimates for one brick by using the translation and isotropic corrections. There was generally very close agreement between the two methods on this data set.

Fig. 4 shows for each animal the superimposed $K$-estimates from all bricks. The theoretical $K$-function for the Poisson process is plotted for comparison. There is a clear dip in the $K$-function over the range $15-35 \mu \mathrm{m}$ for all cases.

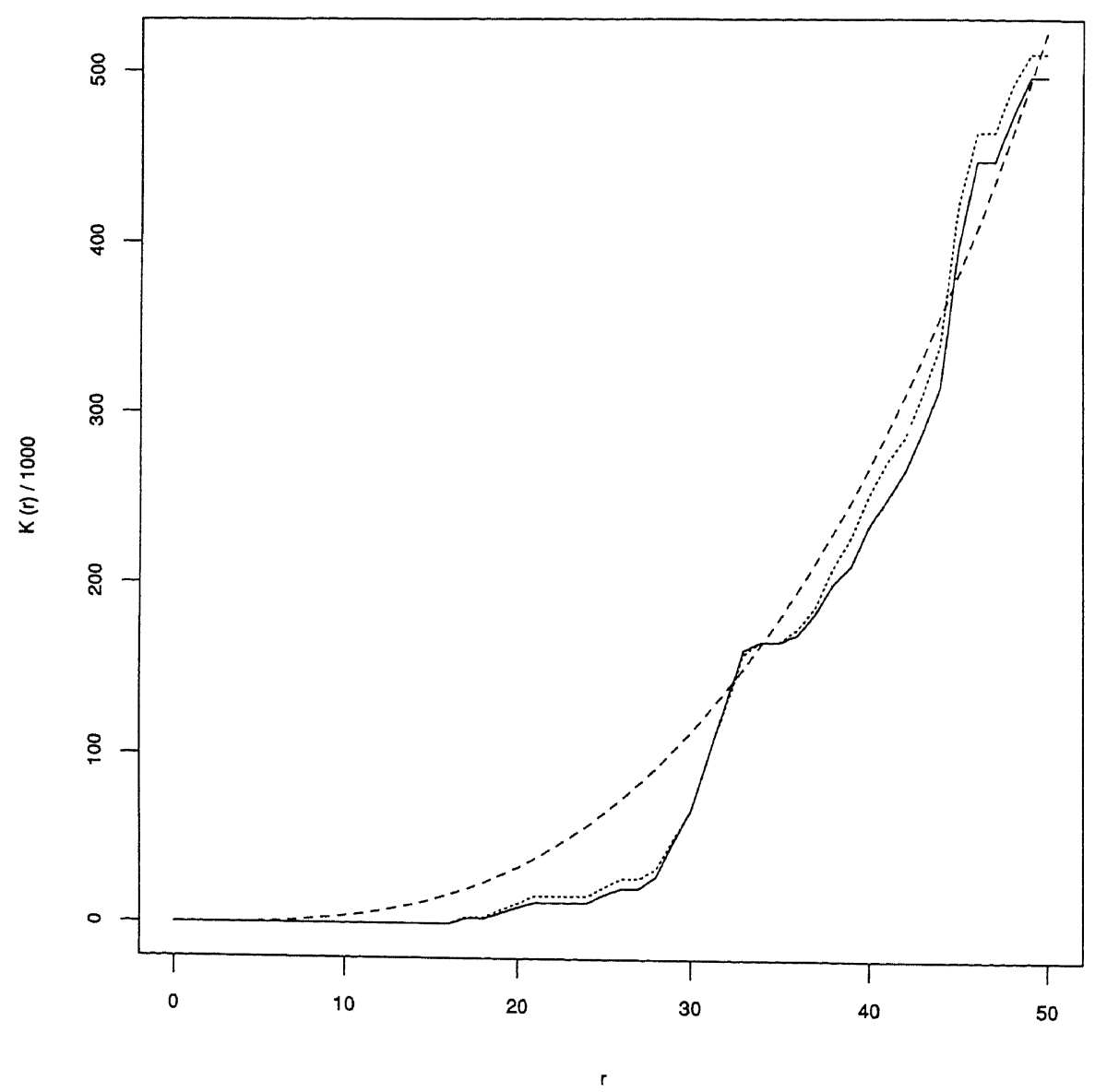

Fig. 3. Comparison of estimates of $K$ by using the Ripley-Hanisch isotropic correction ( - ) and the Miles-Lantuéjoul translation correction $(\cdots \cdots \cdots \cdot)$ for data from brick 1 of animal 4: the theoretical Poisson $K$-function (-------) is plotted for comparison 


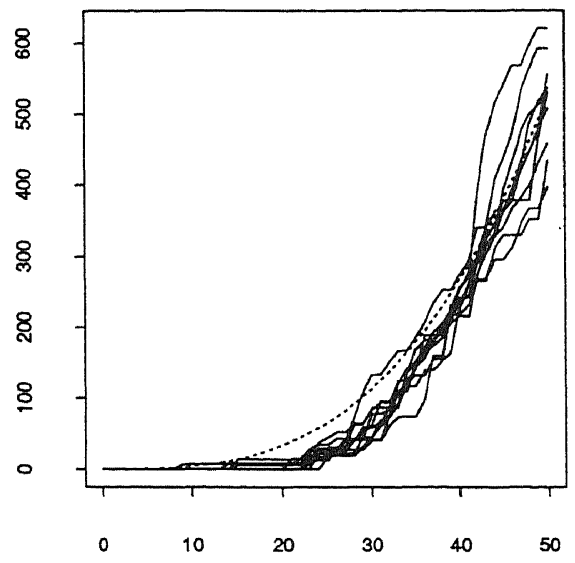

(a)

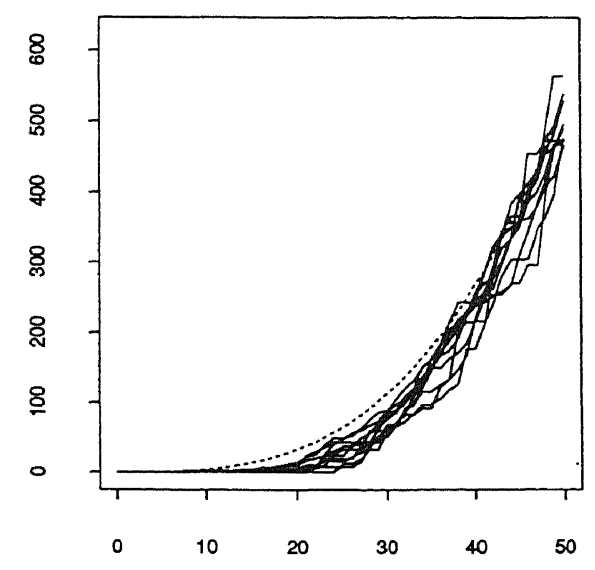

(c)

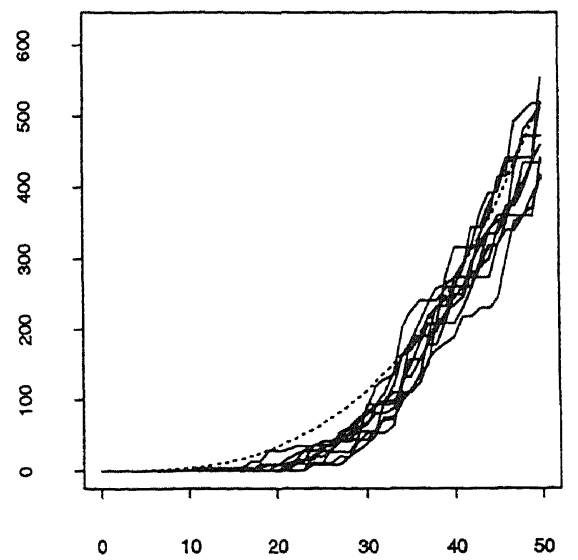

(b)

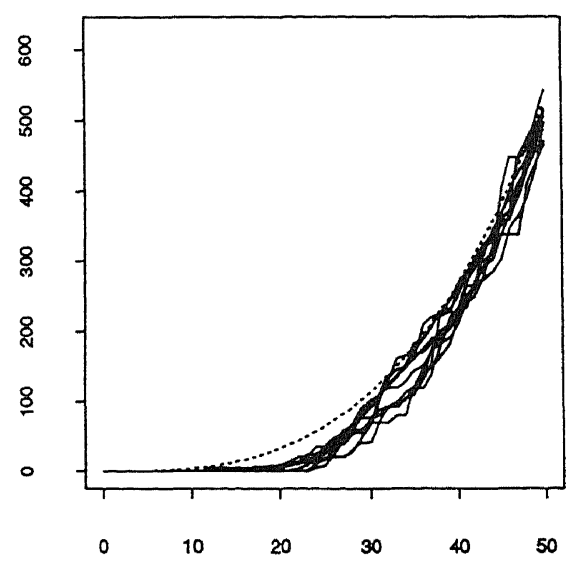

(d)

Fig. 4. Superimposed $K$-function estimates (isotropic correction) from all bricks for (a) animal 1 , (b) animal 2, (c) animal 3 and (d) animal 4

Ripley (1981) recommends that we plot $\sqrt{ } K(r)$ against $r$, because in two dimensions this transformation stabilizes the variance as well as linearizing the plot. Diggle (1983) and others plot the 'residuals' $K(r)-\pi r^{2}$. In three dimensions $\hat{K}^{1 / 3}$ is linear but (contrary to König et al. (1991), p. 416) this does not stabilize the variance. For threedimensional patterns we shall usually plot $K(r)$ against the theoretical Poisson curve $(4 / 3) \pi r^{3}$ in $P-P$ style; Fig. 5.

Fig. 6 shows diagnostic scatterplots of the numerator and denominator of equation (11) for several choices of distance $r$, from all bricks and animals, with numerals identifying the animals. The regression model looks broadly acceptable, although for small $r$ many observations have $U=0$, which might call for revision of the model. Despite the differences previously encountered between animal 1 and the others, there seems to be general agreement in the $K$-functions. 


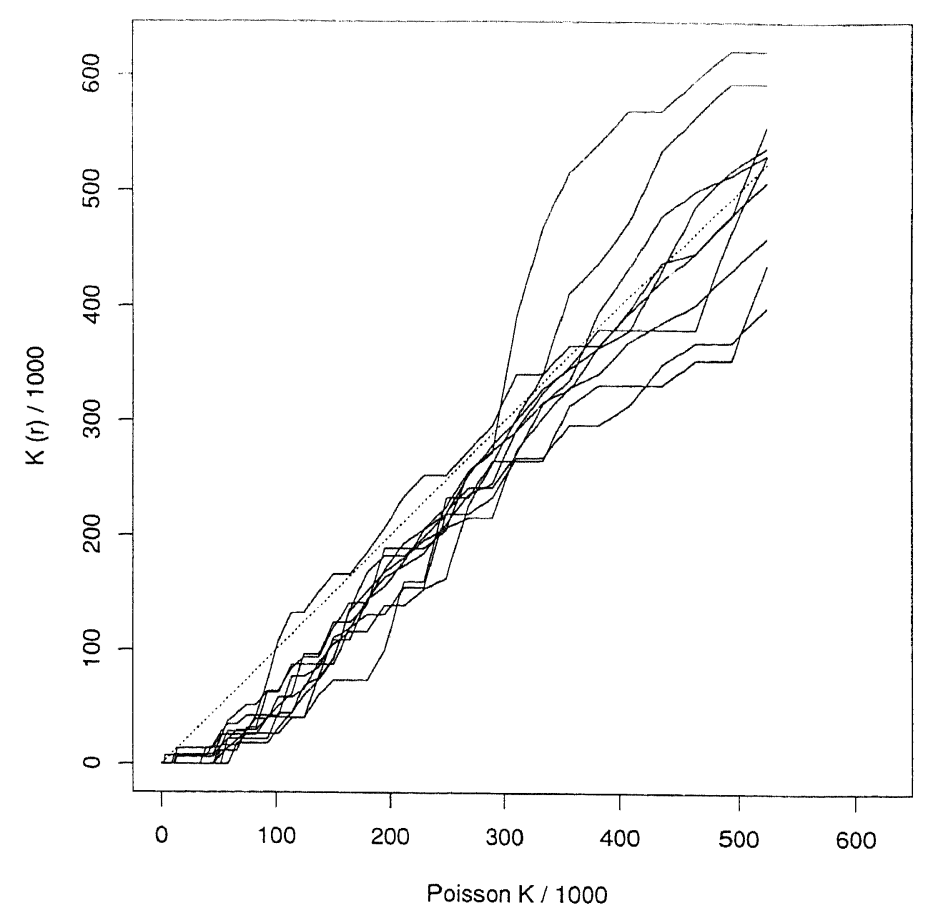

Fig. 5. $K$-statistics (isotropic correction) from each brick in animal 2 plotted $P-P$ style: estimated; $\cdots \cdots \cdot . .$. , Poisson function

Fig. 7 shows pooled $K$-function estimates for each animal, using the ratio regression method to combine replicated bricks. The associated confidence bands are pointwise $95 \%$ confidence intervals computed from the estimated variances under the ratio regression method and the two-tailed $95 \%$ points of the $t$-distribution with 9 degrees of freedom. These figures strengthen the interpretation of a strong dip in the range $15-35 \mu \mathrm{m}$. There is also good agreement between the $K$-functions for the various animals.

Finally Fig. 8 shows an overall $K$-estimate formed by pooling all bricks in animals 2-4 by using the EM approach of Section 5, with pointwise confidence intervals based on the $t_{27}$-distribution.

Our conclusion is that $K$ shows an unambiguous dip in the range $15-35 \mu \mathrm{m}$ and a recovery beyond $35 \mu \mathrm{m}$. This combination suggests an ordered or regular pattern; however, it is not clear how much of the dip can be attributed solely to the absence of overlap between osteocyte lacunae (lacunae were roughly ellipsoidal and typically $10 \mu \mathrm{m} \times 5 \mu \mathrm{m}$ across and $5 \mu \mathrm{m}$ thick, mostly aligned with the main axis in the horizontal plane). Again the recovery at large distances could reflect spatial inhomogeneity at larger scales. These questions could be resolved by simulating a purely inhibitory model.

\subsection{G-function}

Estimators $\hat{G}_{1}$ and $\hat{G}_{3}$ were computed for each brick. Fig. 9 shows a comparison of these for a chosen brick, showing the typical non-monotonicity of $\hat{G}_{1}$. 


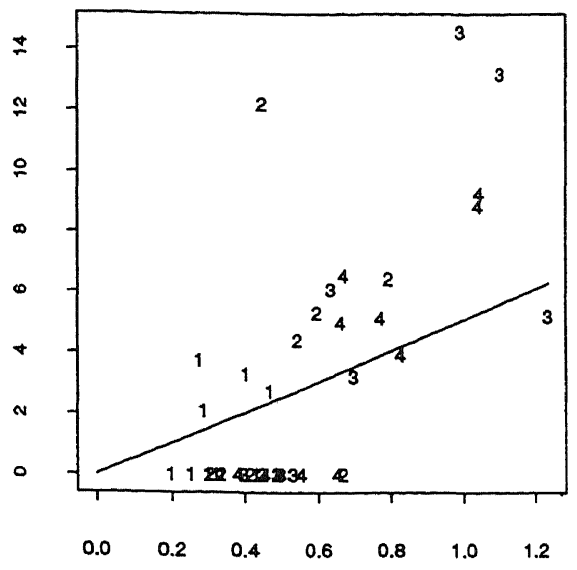

(a)

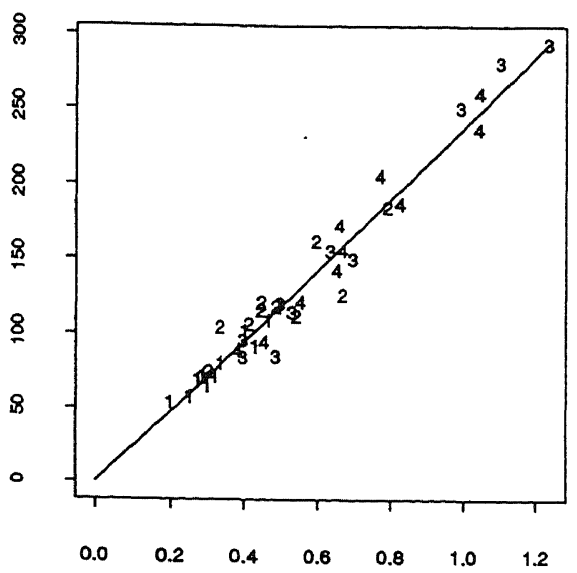

(c)

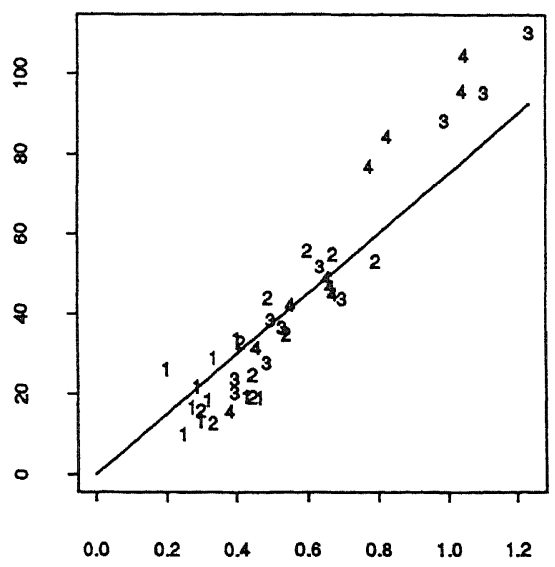

(b)

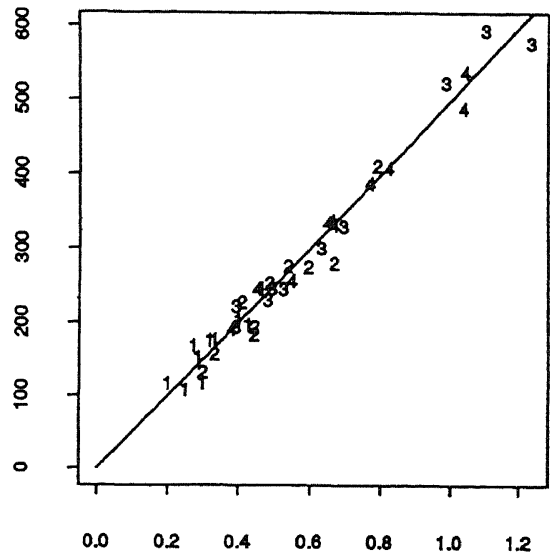

(d)

Fig. 6. Diagnostic scatterplots of the numerator and denominator of equation (11) for (a) $r=20$, (b) $r=30$, (c) $r=40$ and (d) $r=50$ (the data are labelled by animal number)

However, in most cases the individual estimates were absurd, since both the numerator and the denominator consisted of only a few points. Recall that a point contributes to the denominator of $\hat{G}_{3}$ only when it is closer to its nearest neighbour than to the boundary of the box. In three dimensions this condition becomes very stringent. In our data 23 of the 40 bricks had no point $X_{k}$ satisfying the condition. No brick had more than three such points. For the same reason the diagnostic scatterplot for ratio regression was nearly meaningless. The behaviour of $\hat{G}_{1}$ was only slightly better.

Fig. 10 shows pooled $\hat{G}_{1}$-estimates with pointwise confidence intervals, computed by ratio regression. There is moderate evidence supporting an ordered pattern (short nearest neighbour distances are relatively few).

The alternative uncorrected $G$-estimates $G_{B}$ were computed and compared with the corresponding Poisson $G_{B}$-functions for the same bricks, computed numerically by 


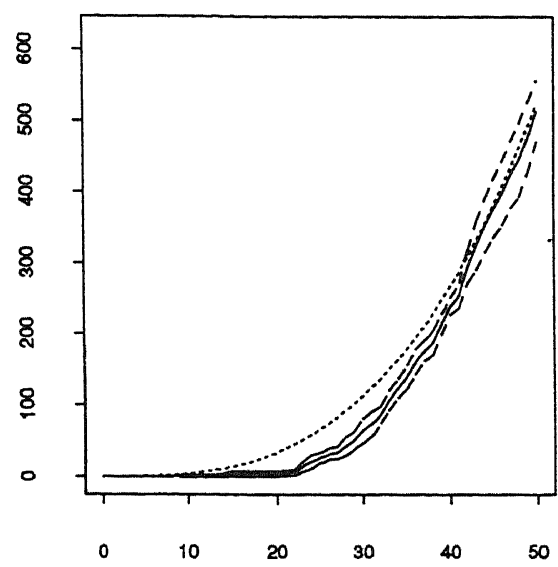

(a)

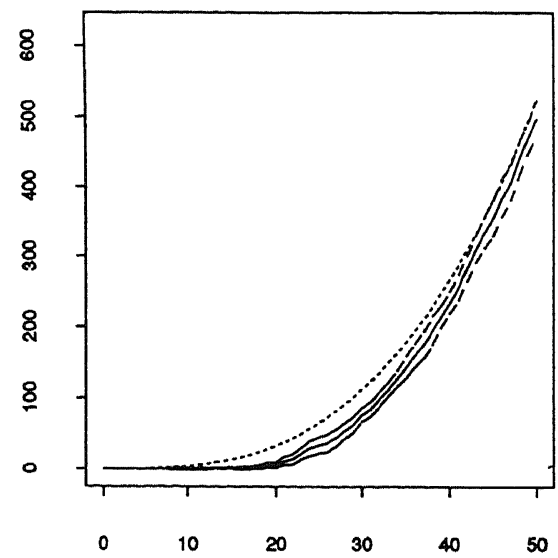

(c)

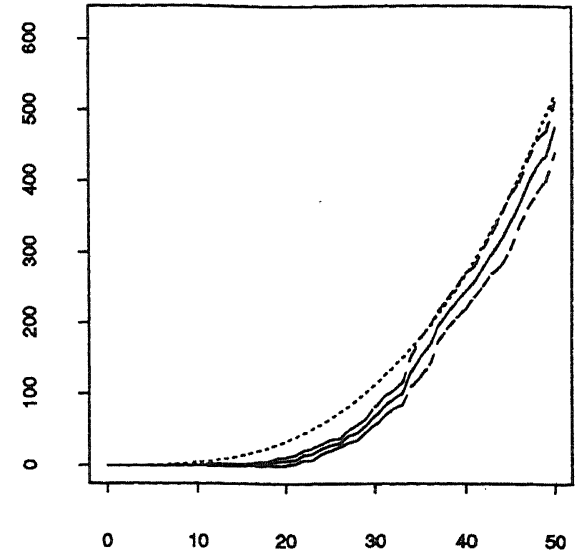

(b)

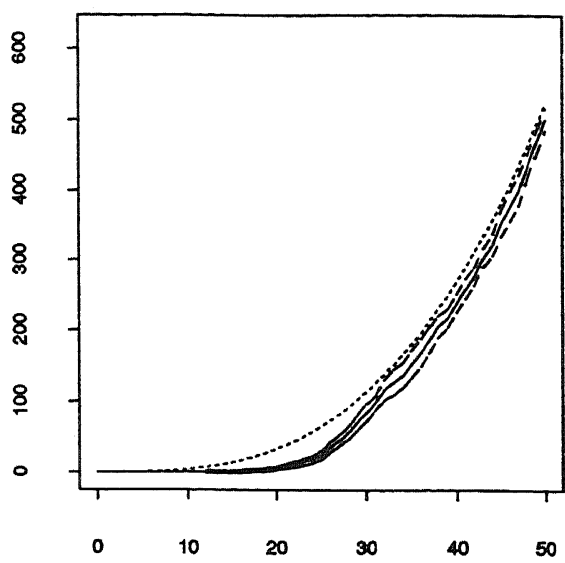

(d)

Fig. 7. $K$-statistics (isotropic correction) pooled across bricks within each animal by using the ratio regression approach of Section 4: (a) animal 1; (b) animal 2; (c) animal 3; (d) animal 4

equation (5). A Monte Carlo test of the Poisson null hypothesis was conducted on each brick independently. 19 simulations of a binomial process (i.e. a fixed number of independent uniform points) were generated in each brick and ranked by mean nearest neighbour distance. The numbers of rejections at the $5 \%$ level (one sided in the direction of larger distances) were 7, 8, 9 and 10 bricks for animals 1, 2, 3 and 4 respectively.

\subsection{F-function}

$F$ was estimated by minus sampling, equation (3). We used the discrete distance transform algorithm of Borgefors $(1984,1986)$ with step distances of 48,58 and 71 , which form a good rational approximation to the proportions $1, \sqrt{2}$ and $\sqrt{3}$. The grid unit or 'voxel' size was $1 \mu \mathrm{m}$. The distance $r(x)$ from each $x$ to the boundary of $B$ was 


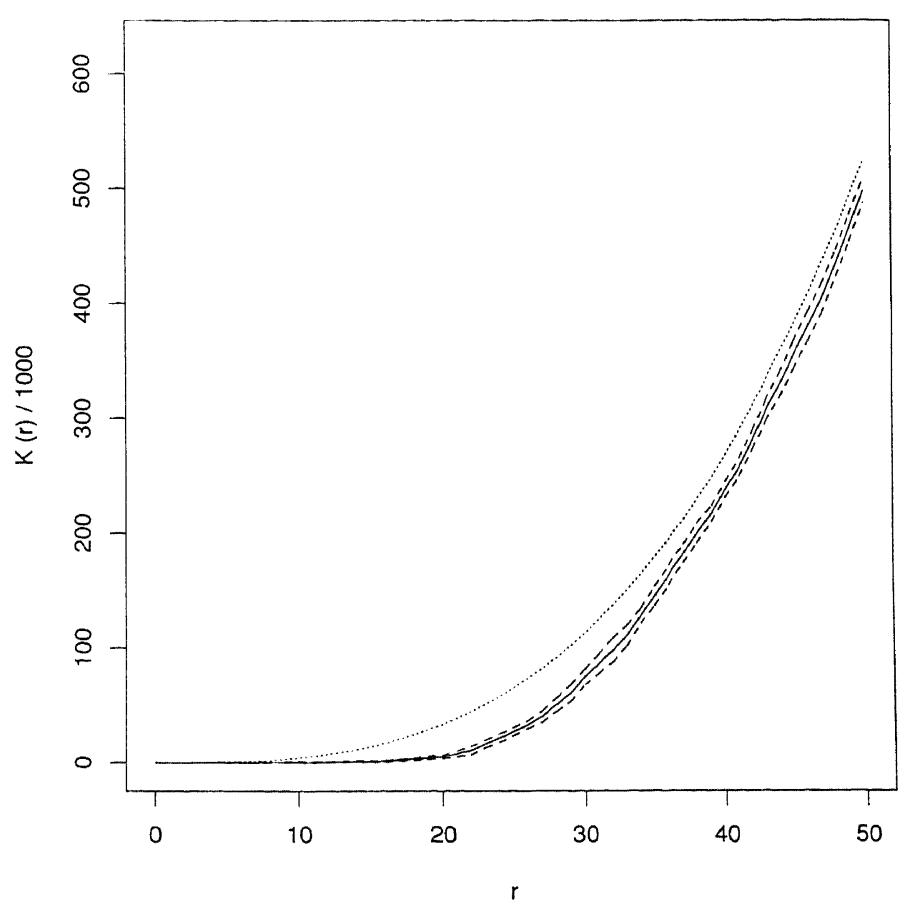

Fig. 8. Grand $K$-statistic (isotropic correction) pooled across all bricks in animals 2-4, using the EM approach of Section 5 (the pointwise $95 \%$ confidence intervals are based on the $t$-distribution with 27 degrees of freedom): __ , estimated; ........, Poisson function; -------, confidence bounds

also computed and the estimate of $F$ was obtained as the cumulative distribution of the distance transform values $d(x)$ for those voxels satisfying $d(x) \leqslant r(x)$.

To compute the theoretical Poisson $F$-function we determined the volume of the sphere of radius $r$ in the discrete distance function, by running the Borgefors algorithm on a larger grid (sufficiently large to include the entire sphere of radius $R$ equal to the maximum diameter of any brick) and taking a cumulative histogram of the result restricted to the range $0-R$.

Fig. 11 shows the pooled estimates for each animal, and Fig. 12 the population estimate pooled from animals 2-4. The Poisson curve lies everywhere outside or on the boundary of the pointwise confidence intervals for these estimates. The plots thus identify a relative shortage of larger empty spaces, again indicative of an ordered pattern.

\section{Discussion}

In this paper we adapted standard estimators for two-dimensional $F-, G$ - and $K$ functions to three dimensions and demonstrated them on a replicated data set. The techniques were successful for $K$ and $F$, but the estimation of $G$ is severely hindered by edge effects and should be studied further. It seems to us that the use of kernel smoothing techniques for $G$ (e.g. König et al. (1991)) only obscures this problem. Some recent work (e.g. Baddeley and Gill (1992)) suggests that a Kaplan-Meier-type estimator of $G$ is more appropriate. 


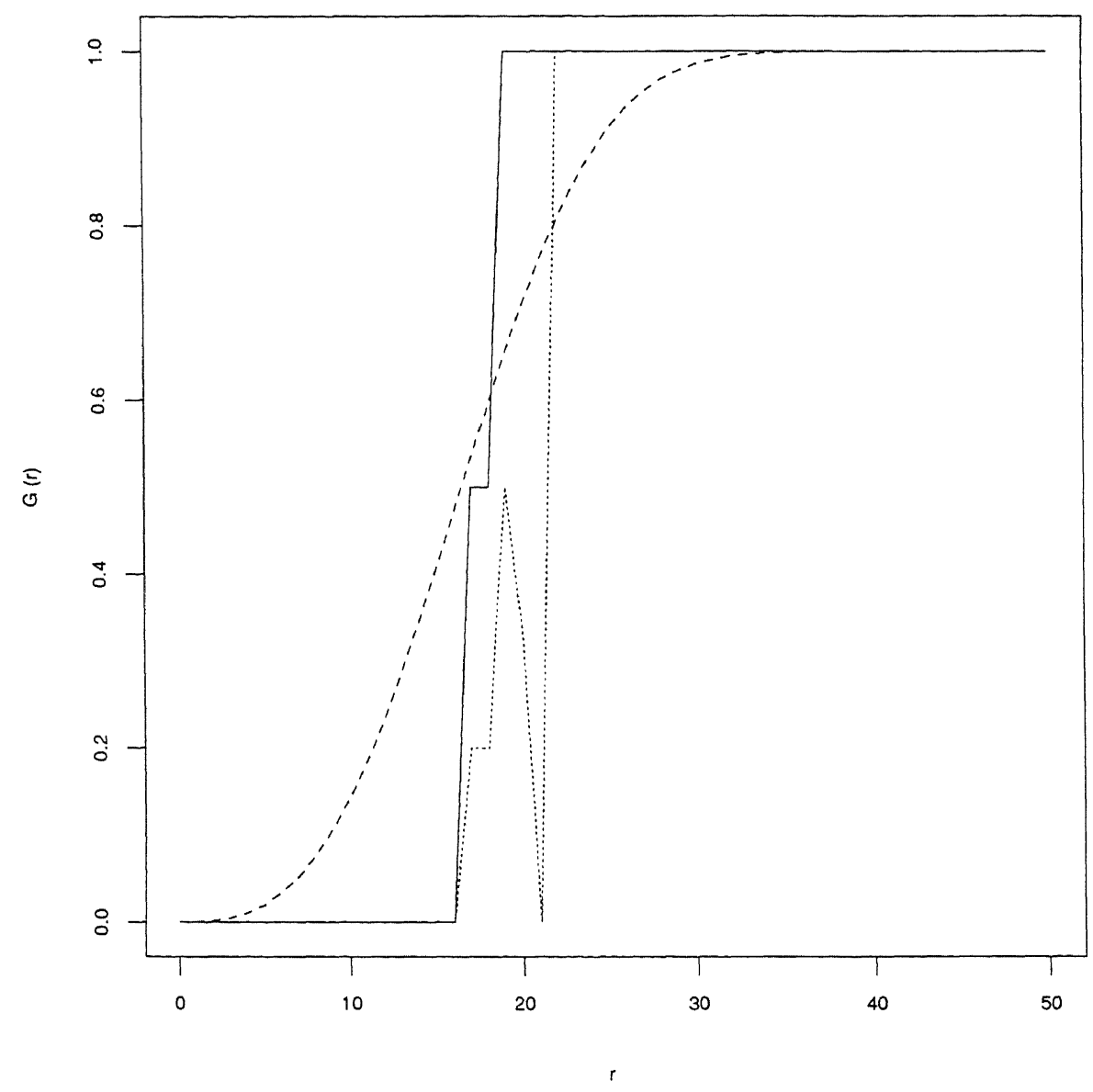

Fig. 9. Comparison of $G$-estimates for brick 1 of animal 4, using the minus sampling correction $(\cdots \cdots \cdots)$ and Hanisch's $G_{3}(-)$ (-------, Poisson function)

The new aspect of replication has been attacked by recognizing that the standard estimators are ratios of unbiased consistent estimators. Variance arguments suggest that the best pooled estimate is the ratio of the mean numerator to the mean denominator; this seems to be a workable approximation, but for edge effects (for large $r$ ) and discrete data effects (for small $r$ ). An alternative approach based on pseudolikelihood models of count data seems worth pursuing.

We have exploited the replication to yield standard errors for the pooled estimates. This is new in spatial statistics, since most previous applications have produced confidence bands around the Poisson theoretical curve rather than confidence intervals around the empirical curve.

A rudimentary analysis of variance has also been developed. Its validity is more difficult to assess from the data studied here, although we managed to detect an unexpected outlier in the animal population.

Monte Carlo tests of the Poisson null hypothesis were generally rejected, more emphatically when based on $F$ than on $G$. This accords with the paucity of data in the 


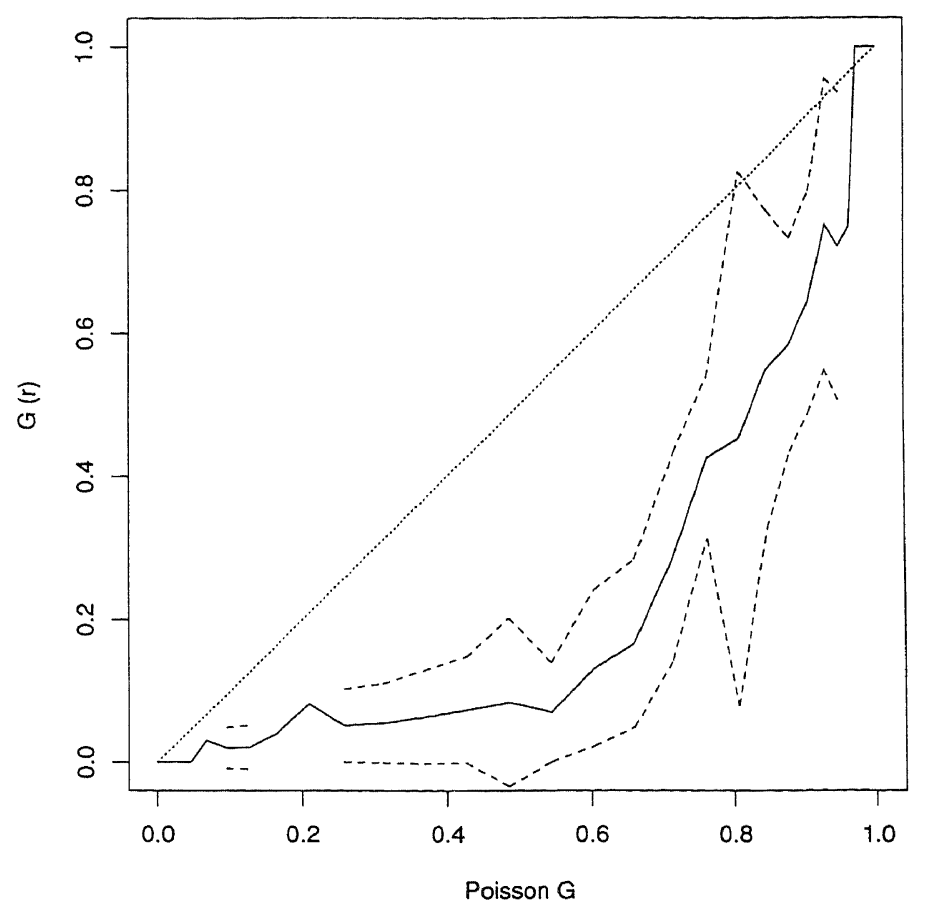

Fig. 10. Grand $G$-statistic (minus sampling correction) pooled across animals 2-4, using the EM approach of Section 5: _ , estimated; $\cdots \cdots \cdots$, Poisson function; -..-.---, pointwise $t_{27} 95 \%$ confidence intervals

numerator and denominator of equations (7) and (8). In two dimensions Diggle (1983) found on the contrary that nearest neighbour distances are more powerful than point to nearest event distances against regular alternatives.

Our conclusions about the data set are that

(a) animal 1 is not from the same population as the others,

(b) there is some evidence of an ordered pattern and

(c) there is appreciable sampling (hence spatial) variation between bricks within an animal.

Regarding the second conclusion, there was widespread agreement between animals $2-4$ in the $K$ - and $F$-functions. All three statistics $F, G$ and $K$ suggested an ordered or regular spatial pattern at a scale of $15-35 \mu \mathrm{m}$. The biological interpretation is uncertain, because this regularity is partly attributable to the physical size of the lacunae (say 5-10 $\mu \mathrm{m}$ across). A similar situation was encountered with the twodimensional biological cell data discussed by Ripley (1981) and Diggle (1983), p. 2. The next step in the analysis of the present data could be to model the process by an inhibitive or regular model such as a Markov point process in the standard way (e.g. Ripley (1988)) to determine how much of the observed regularity can be ascribed to the non-overlap constraint.

For a biologically conclusive analysis we would need to collect three-dimensional images of entire sampling volumes and to fit a random set model. However, the third 


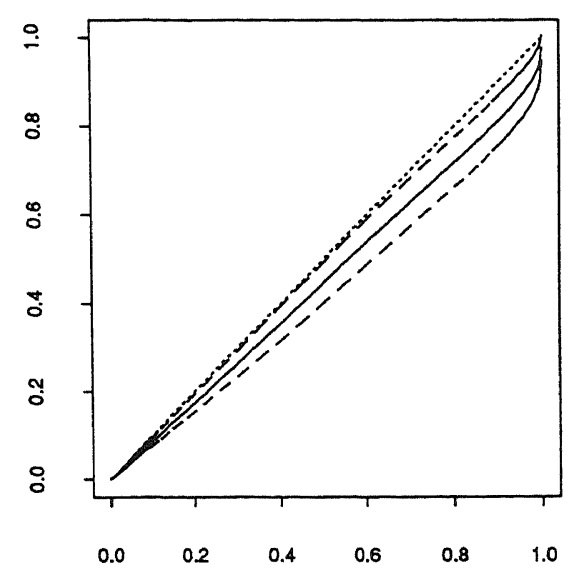

(a)

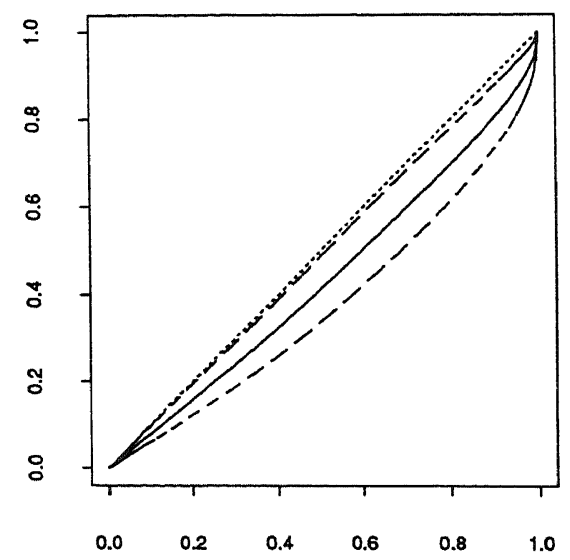

(c)

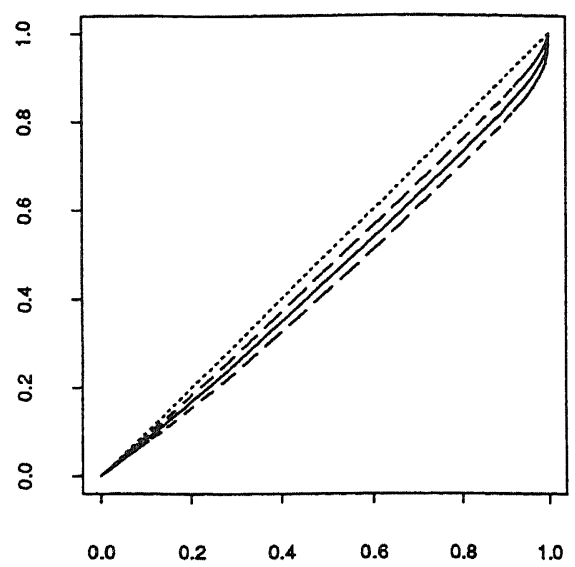

(b)

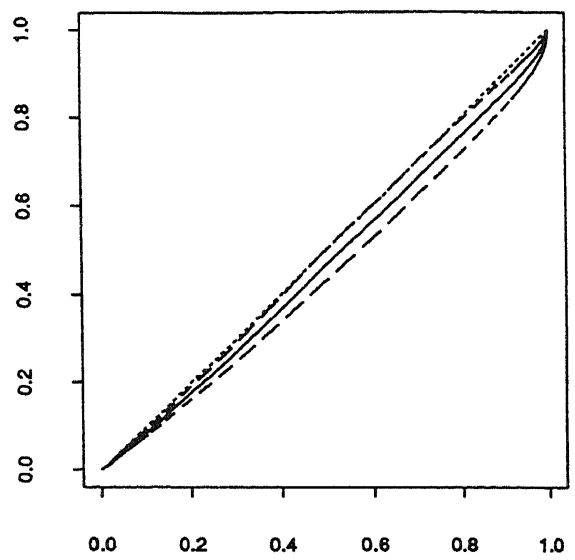

(d)

Fig. 11. F-statistics (minus correction) pooled across bricks by using ratio regression (Section 4): (a) animal 1; (b) animal 2; (c) animal 3; (d) animal 4

finding underlines the fact that a single three-dimensional image would not yield sufficient information because of the spatial sampling variability.

\section{Acknowledgements}

We are particularly grateful to a referee for detailed suggestions. We thank Peter Diggle for comments and prepublication copies of his work, and L. Barendregt, L. M. Cruz Orive, R. D. Gill and M. N. M. van Lieshout for helpful comments. Financial support from Nimbus Records Ltd for the Quantitative 3-D Microscopy Research Group, University of Liverpool, is gratefully acknowledged.

\section{Appendix A: Sphere Volume and Surface Area}

The purpose of this appendix is to derive expressions for the volume and surface area of the intersection between a three-dimensional rectangular box 


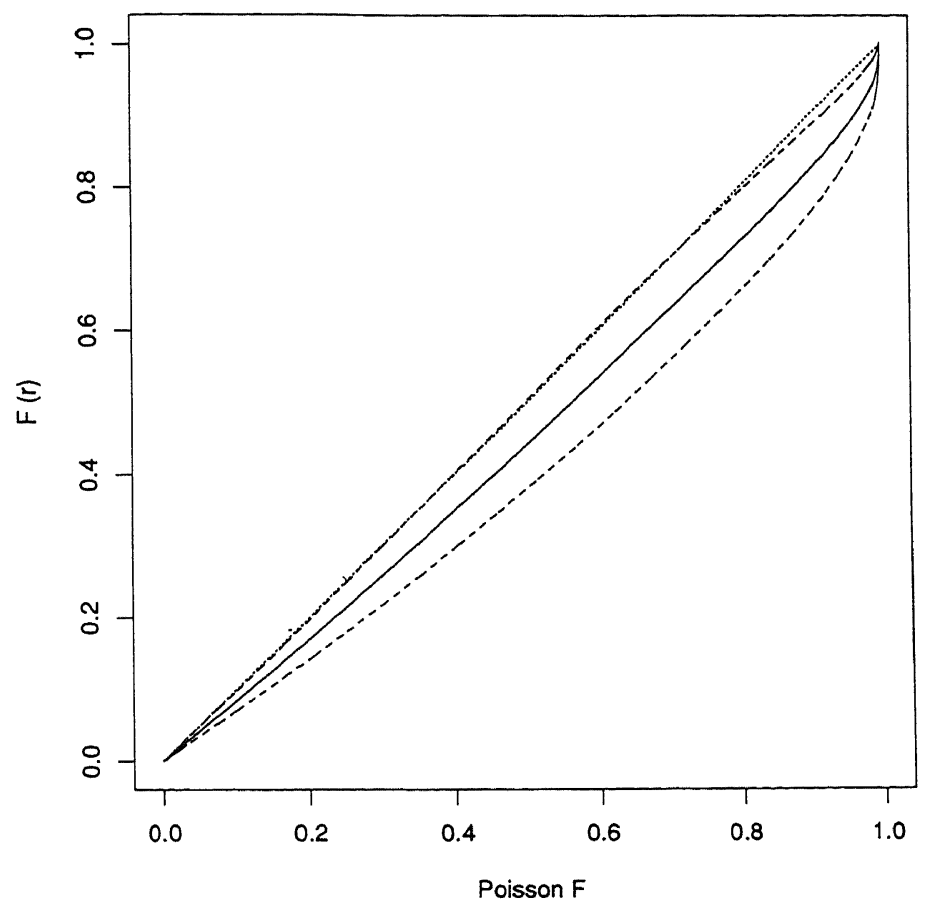

Fig. 12. Grand $F$-estimate (minus correction) pooled across animals $2-4$ by using the EM technique (Section 5): - - , estimated; …....., Poisson function; -------, pointwise $95 \%$ confidence intervals

$$
B=\left\{\mathrm{x} \in \mathbf{R}^{3}: 0 \leqslant x_{i}<b_{i}, i=1,2,3\right\}
$$

with sides $b_{i}>0$ and a sphere

$$
S(\mathbf{x}, r)=\left\{\mathbf{y} \in \mathbf{R}^{3}:\|\mathbf{y}-\mathbf{x}\| \leqslant r\right\}
$$

with centre $\mathbf{x} \in B$ and radius $r>0$. The results are equations (27), (28), (33) and (34) for volume, and equations (35) and (37) for area.

Thus the aim is to compute

$$
\begin{gathered}
V(\mathbf{x}, r)=\mathscr{L}^{3}\{B \cap S(\mathbf{x}, r)\}, \\
A(\mathbf{x}, r)=\mathscr{H}^{2}\{B \cap \partial S(\mathbf{x}, r)\}
\end{gathered}
$$

where $\mathscr{L}^{3}$ is Lebesgue volume measure and $\mathscr{H}^{2}$ is two-dimensional Hausdorff (surface area) measure in $\mathbf{R}^{3}$. Representing $B$ as an intersection of half-spaces

$$
B=H_{1}(0) \cap H_{1}\left(b_{1}\right)^{c} \cap H_{2}(0) \cap H_{2}\left(b_{2}\right)^{c} \cap H_{3}(0) \cap H_{3}\left(b_{3}\right)^{c}
$$

where $H_{i}(t)=\left\{x \in \mathbf{R}^{3}: x_{i} \geqslant t\right\}$, and applying the inclusion-exclusion formula, we obtain

$$
\begin{aligned}
\frac{4}{3} \pi r^{3}-V(\mathbf{x}, r)= & \sum_{i=1}^{3}\left\{V_{1}\left(x_{i}, r\right)+V_{1}\left(b_{i}-x_{i}, r\right)\right\}-\sum \sum_{i<j}\left\{V_{2}\left(x_{i}, x_{j}, r\right)+V_{2}\left(x_{i}, b_{j}-x_{j}, r\right)\right. \\
& \left.+V_{2}\left(b_{i}-x_{i}, x_{j}, r\right)+V_{2}\left(b_{i}-x_{i}, b_{j}-x_{j}, r\right)\right\}+V_{3}\left(x_{1}, x_{2}, x_{3}, r\right) \\
& +V_{3}\left(x_{1}, x_{2}, b_{3}-x_{3}, r\right)+V_{3}\left(x_{1}, b_{2}-x_{2}, x_{3}, r\right)+V_{3}\left(x_{1}, b_{2}-x_{2}, b_{3}-x_{3}, r\right) \\
& +V_{3}\left(b_{1}-x_{1}, x_{2}, x_{3}, r\right)+V_{3}\left(b_{1}-x_{1}, x_{2}, b_{3}-x_{3}, r\right)+V_{3}\left(b_{1}-x_{1}, b_{2}-x_{2}, x_{3}, r\right) \\
& +V_{3}\left(b_{1}-x_{1}, b_{2}-x_{2}, b_{3}-x_{3}, r\right)
\end{aligned}
$$


where $V_{1}, V_{2}$ and $V_{3}$ represent the volumes delimited by 1,2 or 3 half-spaces:

$$
\begin{gathered}
V_{1}\left(t_{1}, r\right)=\mathscr{L}^{3}\left\{S(0, r) \cap H_{1}\left(t_{1}\right)\right\}, \\
V_{2}\left(t_{1}, t_{2}, r\right)=\mathscr{L}^{3}\left\{S(0, r) \cap H_{1}\left(t_{1}\right) \cap H_{2}\left(t_{2}\right)\right\}, \\
V_{3}\left(t_{1}, t_{2}, t_{3}, r\right)=\mathscr{L}^{3}\left\{S(0, r) \cap H_{1}\left(t_{1}\right) \cap H_{2}\left(t_{2}\right) \cap H_{3}\left(t_{3}\right)\right\}
\end{gathered}
$$

for $t_{i} \geqslant 0$. If we define $U(a, b, c)=V_{3}(a, b, c, 1)$ then by symmetry and scaling properties of volume

$$
\left.\begin{array}{c}
V_{1}\left(t_{1}, r\right)=r^{3} V_{1}\left(t_{1} / r, 1\right)=4 r^{3} U\left(t_{1} / r, 0,0\right), \\
V_{2}\left(t_{1}, t_{2}, r\right)=r^{3} V_{2}\left(t_{1} / r, t_{2} / r, 1\right)=2 r^{3} U\left(t_{1} / r, t_{2} / r, 0\right), \\
V_{3}\left(t_{1}, t_{2}, t_{3}, r\right)=r^{3} U\left(t_{1} / r, t_{2} / r, t_{3} / r\right) .
\end{array}\right\}
$$

It suffices to find an expression for $U(a, b, c)$ when $a, b, c \geqslant 0$ and $a^{2}+b^{2}+c^{2}<1$. Now

$$
\begin{aligned}
U(a, b, c) & =\int_{c}^{\left(1-a^{2}-b^{2}\right)^{1 / 2}} \int_{b}^{\left(1-z^{2}-a^{2}\right)^{1 / 2}} \int_{c}^{\left(1-z^{2}-y^{2}\right)^{1 / 2}} \mathrm{~d} x \mathrm{~d} y \mathrm{~d} z \\
& =\int_{c}^{\left(1-a^{2}-b^{2}\right)^{1 / 2}} \int_{b}^{\left(1-z^{2}-a^{2}\right)^{1 / 2}}\left\{\left(1-z^{2}-y^{2}\right)^{1 / 2}-c\right\} \mathrm{d} y \mathrm{~d} z .
\end{aligned}
$$

Using

$$
\int\left(1-a^{2}-x^{2}\right)^{1 / 2} \mathrm{~d} x=\frac{1}{2} x\left(1-a^{2}-x^{2}\right)^{1 / 2}+\frac{1}{2}\left(1-a^{2}\right) \sin ^{-1}\left\{\frac{x}{\left(1-a^{2}\right)^{1 / 2}}\right\}
$$

this becomes

$$
\begin{aligned}
U(a, b, c)= & \frac{1}{2} a b\left(1-a^{2}-b^{2}\right)^{1 / 2}+\frac{1}{4} a c\left(1-a^{2}-c^{2}\right)^{1 / 2}+\frac{1}{4} b c\left(1-b^{2}-c^{2}\right)^{1 / 2} \\
& -\frac{1}{4} a\left(1-a^{2}\right)\left[\frac{\pi}{2}-\sin ^{-1}\left\{\frac{b}{\left(1-a^{2}\right)^{1 / 2}}\right\}-\sin ^{-1}\left\{\frac{c}{\left(1-a^{2}\right)^{1 / 2}}\right\}\right] \\
& -\frac{1}{4} b\left(1-b^{2}\right)\left[\frac{\pi}{2}-\sin ^{-1}\left\{\frac{a}{\left(1-b^{2}\right)^{1 / 2}}\right\}-\sin ^{-1}\left\{\frac{c}{\left(1-b^{2}\right)^{1 / 2}}\right\}\right] \\
& +\frac{\pi}{4}\left(1-a^{2}-b^{2}\right)^{1 / 2}-\frac{\pi}{4} c-\frac{\pi}{12}\left(1-a^{2}-b^{2}\right)^{3 / 2}+\frac{\pi}{12} c^{3} \\
& -\frac{1}{2} \int_{c}^{\left(1-a^{2}-b^{2}\right)^{1 / 2}}\left(1-z^{2}\right) \sin ^{-1}\left\{\frac{a}{\left(1-z^{2}\right)^{1 / 2}}\right\} \mathrm{d} z \\
& -\frac{1}{2} \int_{c}^{\left(1-a^{2}-b^{2}\right)^{1 / 2}}\left(1-z^{2}\right) \sin ^{-1}\left\{\frac{b}{\left(1-z^{2}\right)^{1 / 2}}\right\} \mathrm{d} z .
\end{aligned}
$$

Here the inverse sine maps $(0,1)$ to $(0, \pi / 2)$. The remaining terms are integrated by parts with the help of the following identities, holding for $0<A<1,0<t<\left(1-A^{2}\right)^{1 / 2}$ : 


$$
\begin{gathered}
\int_{0}^{t}\left(1-A^{2}-x^{2}\right)^{-1 / 2} \mathrm{~d} x=\sin ^{-1}\left\{\frac{t}{\left(1-A^{2}\right)^{1 / 2}}\right\} ; \\
\int_{0}^{t}\left(1-x^{2}\right)^{-1}\left(1-A^{2}-x^{2}\right)^{-1 / 2} \mathrm{~d} x=A^{-1} \tan ^{-1}\left\{\frac{A t}{\left(1-A^{2}-t^{2}\right)^{1 / 2}}\right\} ; \\
\int_{0}^{t} x^{2}\left(1-A^{2}-x^{2}\right)^{-1 / 2} \mathrm{~d} x=\frac{1}{2}\left(1-A^{2}\right) \sin ^{-1}\left\{\frac{t}{\left(1-A^{2}\right)^{1 / 2}}\right\}-\frac{1}{2} t\left(1-A^{2}-t^{2}\right)^{1 / 2} \\
\int_{0}^{t}\left(1-x^{2}\right)^{-1}\left(1-A^{2}-x^{2}\right)^{-1 / 2} \mathrm{~d} x=\left(1-A^{2}\right)^{-1 / 2} \tan ^{-1}\left\{\frac{t}{\left(1-A^{2}-t^{2}\right)^{1 / 2}}\right\} \\
-A\left(1-A^{2}\right)^{-1 / 2} \tan ^{-1}\left\{\frac{A t}{\left(1-A^{2}-t^{2}\right)^{1 / 2}}\right\} .
\end{gathered}
$$

The last three results were suggested by applying the complex identity $\log (a+\mathrm{i} b)=$ $\log \left(a^{2}+b^{2}\right)^{1 / 2}+\mathrm{i} \tan ^{-1}(b / a)$ to Gradshteyn and Ryzhik's (1980) formulae (69) and (8) in section 2.584 and (33) in section 2.583 . They can be checked directly.

Collecting terms and applying trigonometric identities between inverse sine and inverse tangent, we finally have

$$
U(a, b, c)=\frac{\pi}{12}\left\{2-3(a+b+c)+\left(a^{3}+b^{3}+c^{3}\right)\right\}+W(a, b)+W(b, c)+W(a, c)-a b c
$$

where

$$
\begin{aligned}
W(x, y)= & \left(\frac{1}{2} x-\frac{1}{6} x^{3}\right) \tan ^{-1}\left\{\frac{y}{\left(1-x^{2}-y^{2}\right)^{1 / 2}}\right\}+\left(\frac{1}{2} y-\frac{1}{6} y^{3}\right) \tan ^{-1}\left\{\frac{x}{\left(1-y^{2}-x^{2}\right)^{1 / 2}}\right\} \\
& -\frac{1}{3} \tan ^{-1}\left\{\frac{x y}{\left(1-x^{2}-y^{2}\right)^{1 / 2}}\right\}+\frac{1}{3} x y\left(1-x^{2}-y^{2}\right)^{1 / 2} .
\end{aligned}
$$

The values of the inverse tangent computed here lie in $[0, \pi / 2)$. As a check we can verify the boundary conditions

$$
\begin{gathered}
U(0,0,0)=\frac{\pi}{6}, \\
U(a, 0,0)=\frac{\pi}{12}\left(2-3 a+a^{3}\right) .
\end{gathered}
$$

Thus formulae (27), (28), (33) and (34) specify an algorithm for computing $V(\mathbf{x}, r)$.

Turning to the surface area problem, we have a decomposition that is analogous to equation (27) with $A(\mathbf{x}, r)$ replacing $V(\mathbf{x}, r), A_{1}, A_{2}$ and $A_{3}$ replacing $V_{1}, V_{2}$ and $V_{3}$ and $4 \pi r^{2}$ replacing $\frac{4}{3} \pi r^{3}$. Analogous to system (28) we have

$$
\left.\begin{array}{c}
A_{1}\left(t_{1}, r\right)=4 r^{2} C\left(t_{1} / r, 0,0\right), \\
A_{2}\left(t_{1}, t_{2}, r\right)=2 r^{2} C\left(t_{1} / r, t_{2} / r, 0\right), \\
A_{3}\left(t_{1}, t_{2}, t_{3}, r\right)=r^{2} C\left(t_{1} / r, t_{2} / r, t_{2} / r\right) .
\end{array}\right\}
$$

To compute $C(a, b, c)$ for $a, b, c>0, a^{2}+b^{2}+c^{2}<1$, parameterize the unit sphere $S(0,1)$ by angular co-ordinates $\theta$ and $\phi$ defined by 


$$
\begin{gathered}
u:[0,2 \pi) \times\left(-\frac{\pi}{2}, \frac{\pi}{2}\right) \rightarrow S(0,1), \\
u(\theta, \phi)=(\cos \theta \cos \phi, \sin \theta \cos \phi, \sin \phi),
\end{gathered}
$$

i.e. $\theta$ represents 'longitude' and $\phi$ is 'latitude'. The representation of area measure is now

$$
u_{\#} \mathrm{~d} S=\cos \phi \mathrm{d} \theta \wedge \mathrm{d} \phi .
$$

Let $T=S(0,1) \cap H_{1}(a) \cap H_{2}(b) \cap H_{3}(c)$ where $a, b, c>0$. Thus $C(a, b, c)=\mathscr{C}^{2}(T)$ and

$$
\begin{aligned}
u^{-1}(T)= & \{(\theta, \phi): \cos \theta \cos \phi \geqslant a, \sin \theta \cos \phi \geqslant b, \sin \phi \geqslant c\} \\
= & \left\{(\theta, \phi): \sin ^{-1}\left\{\frac{b}{\sqrt{ }\left(1-c^{2}\right)}\right\} \leqslant \theta \leqslant \cos ^{-1}\left\{\frac{a}{\sqrt{ }\left(1-c^{2}\right)}\right\},\right. \\
& \left.\sin ^{-1} c \leqslant \phi \leqslant \cos ^{-1}\left\{\max \left(\frac{a}{\cos \theta}, \frac{b}{\sin \theta}\right)\right\}\right\}
\end{aligned}
$$

where henceforth $0<\theta, \phi<\pi / 2$. We have

$$
\begin{aligned}
& C(a, b, c)=\mathscr{H}^{2}(T) \\
& =\int_{\sin ^{-1}\left\{b / \sqrt{ }\left(1-c^{2}\right)\right\}}^{\cos ^{-1}\left\{a / \sqrt{ }\left(1-c^{2}\right)\right\}} \int_{\sin ^{-1} c}^{\cos ^{-1}\{\max (a / \cos \theta, b / \sin \theta)\}} \cos \phi \mathrm{d} \phi \mathrm{d} \theta \\
& =\int_{\sin ^{-1}\left\{b / \sqrt{ }\left(1-c^{2}\right)\right\}}^{\tan ^{-1}(b / a)} \int_{\sin ^{-1} c}^{\cos ^{-1}(b / \sin \theta)} \cos \phi \mathrm{d} \phi \mathrm{d} \theta+\int_{\tan ^{-1}(b / a)}^{\cos ^{-1}\left\{a / \sqrt{\left.\left(1-c^{2}\right)\right\}}\right.} \int_{\sin ^{-1} c}^{\cos ^{-1}(a / \cos \theta)} \cos \phi \mathrm{d} \phi \mathrm{d} \theta
\end{aligned}
$$

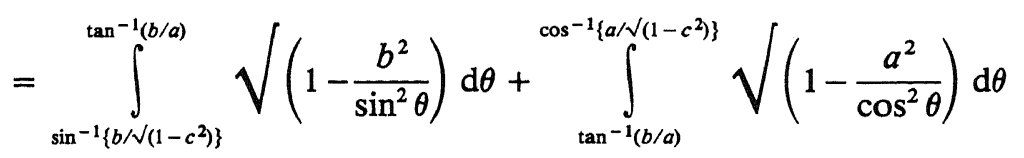

$$
\begin{aligned}
& +c\left[\sin ^{-1}\left\{\frac{b}{\sqrt{\left(1-c^{2}\right)}}\right\}-\cos ^{-1}\left\{\frac{a}{\sqrt{ }\left(1-c^{2}\right)}\right\}\right] \\
& =I\left[\cos ^{-1}\left\{\frac{a}{\sqrt{\left(1-c^{2}\right)}}\right\}, a\right]-I\left\{\tan ^{-1}\left(\frac{b}{a}\right), a\right\} \\
& +I\left[\cos ^{-1}\left\{\frac{b}{\sqrt{ }\left(1-c^{2}\right)}\right\}, b\right]-I\left\{\tan ^{-1}\left(\frac{a}{b}\right), b\right\} \\
& +c\left[\sin ^{-1}\left\{\frac{b}{\sqrt{\left(1-c^{2}\right)}}\right\}-\cos ^{-1}\left\{\frac{a}{\sqrt{\left(1-c^{2}\right)}}\right\}\right]
\end{aligned}
$$

where the range of the inverse tangent is $(-\pi / 2, \pi / 2)$ and

$$
I(t, A)=\int_{0}^{\sin t} \frac{\sqrt{ }\left(1-A^{2}-x^{2}\right)}{1-x^{2}} \mathrm{~d} x
$$

for $0<A<1,0<t<\sin ^{-1}\left(1-A^{2}\right)^{1 / 2}$. Substituting equation (32) we finally obtain 


$$
\begin{aligned}
C(a, b, c)= & \tan ^{-1}\left\{\frac{\left(1-a^{2}-c^{2}\right)^{1 / 2}}{a c}\right\}+\tan ^{-1}\left\{\frac{\left(1-b^{2}-c^{2}\right)^{1 / 2}}{b c}\right\}+\tan ^{-1}\left\{\frac{\left(1-a^{2}-b^{2}\right)^{1 / 2}}{a b}\right\} \\
& -a \tan ^{-1}\left\{\frac{\left(1-a^{2}-c^{2}\right)^{1 / 2}}{a b}\right\}+a \tan ^{-1}\left\{\frac{b}{\left(1-a^{2}-b^{2}\right)^{1 / 2}}\right\}-b \tan ^{-1}\left\{\frac{\left(1-b^{2}-c^{2}\right)^{1 / 2}}{c}\right\} \\
& +b \tan ^{-1}\left\{\frac{a}{\left(1-a^{2}-b^{2}\right)^{1 / 2}}\right\}-c \tan ^{-1}\left\{\frac{\left(1-a^{2}-c^{2}\right)^{1 / 2}}{a}\right\} \\
& +c \tan ^{-1}\left\{\frac{b}{\left(1-a^{2}-b^{2}\right)^{1 / 2}}\right\}-\pi
\end{aligned}
$$

when $a^{2}+b^{2}+c^{2}<1$, and $C(a, b, c)=0$ otherwise. Here the values of the inverse tangent lie in $(0, \pi / 2)$. As a check, one can verify the boundary conditions

$$
\begin{gathered}
C(u, 0,0)=C(0, u, 0)=C(0,0, u)=\pi(1-u) / 2, \\
C(1 / \sqrt{3}, 1 / \sqrt{3}, 1 / \sqrt{3})=0 .
\end{gathered}
$$

Thus equations (35) and (37) specify an algorithm for computing the surface area $A(\mathbf{x}, r)$ and the edge-effect correction factor

$$
w(\mathbf{x}, r)=A(\mathbf{x}, r) / 4 \pi r^{2} .
$$

(We thank Dr C. C. Taylor, University of Leeds, for the suggestion leading to equations (30)-(32).)

\section{References}

Baddeley, A. J. (1980) A limit theorem for statistics of spatial data. Adv. Appl. Probab., 12, 447-461.

Baddeley, A. J. and Gill, R. D. (1992) Kaplan-Meier estimators for interpoint distance distributions of spatial point processes. Research Report 718. Mathematical Institute, University of Utrecht, Utrecht.

Baddeley, A. J., Howard, C. V., Boyde, A. and Reid, S. (1987) Three-dimensional analysis of the spatial distribution of particles using the tandem-scanning reflected light microscope. Acta Ster., 6, suppl. II, 87-100.

Baddeley, A. J. and Silverman, B. W. (1984) A cautionary example on the use of second-order methods for analyzing point patterns. Biometrics, 40, 1089-1094.

Bjaalie, J. G. and Diggle, P. J. (1990) Statistical analysis of corticopontine neuron distribution in visual areas 17, 18 and 19 of the cat. J. Comp. Neurol., 295, 15-32.

Borgefors, G. (1984) Distance transformations in arbitrary dimensions. Comput. Vis. Graph. Image Process., 27, 321-345.

(1986) Distance transformations in digital images. Comput. Vis. Graph. Image Process., 34, 344-371.

Braendgaard, H. and Gundersen, H. J. G. (1986) The impact of recent stereological advances on quantitative studies of the nervous system. J. Neurosci. Meth., 18, 39-78.

Cochran, W. G. (1977) Sampling Techniques, 3rd edn. New York: Wiley.

Cox, D. R. and Isham, V. (1980) Point Processes. London: Chapman and Hall.

Cressie, N. A. C. (1991) Statistics for Spatial Data. New York: Wiley.

Cruz Orive, L.-M. (1980) Best linear unbiased estimators for stereology. Biometrics, 36, 595-605.

Daley, D. J. and Vere-Jones, D. (1989) Introduction to the Theory of Point Processes. Heidelberg: Springer.

Diggle, P. J. (1983) Statistical Analysis of Spatial Point Patterns. London: Academic Press.

Diggle, P. J. and Gratton, R. J. (1984) Monte Carlo methods of inference for implicit statistical models (with discussion). J. R. Statist. Soc. B, 46, 193-227.

Diggle, P. J., Lange, N. and Benes, F. M. (1991) Analysis of variance for replicated spatial point patterns in clinical neuroanatomy. J. Am. Statist. Ass., 86, 618-625.

Diggle, P. J. and Matérn, B. (1981) On sampling designs for the estimation of point-event nearest neighbour distributions. Scand. J. Statist., 7, 80-84. 
Gradshteyn, I. S. and Ryzhik, I. M. (1980) Table of Integrals, Series and Products. New York: Academic Press.

Hall, P. (1988) An Introduction to the Theory of Coverage Processes. New York: Wiley.

Hanisch, K.-H. (1984) Some remarks on estimators of the distribution function of nearest neighbour distance in stationary spatial point patterns. Math. Operforsch. Statist. Ser. Statist., 15, 409-412.

Howard, C. V., Reid, S., Baddeley, A. J. and Boyde, A. (1985) Unbiased estimation of particle density in the tandem-scanning reflected light microscope. J. Microsc., 138, 203-212.

Jolivet, E. (1980) Central limit theorem and convergence of empirical processes for stationary point processes. In Point Processes and Queueing Problems (eds P. Bastfai and J. Tomko), pp. 117-161. Amsterdam: North-Holland.

König, D., Carvajal-Gonzalez, S., Downs, A. M., Vassy, J. and Rigaut, J. P. (1991) Modelling and analysis of 3-d arrangements of particles by point processes with examples of application to biological data obtained by confocal scanning light microscopy. J. Microsc., 161, 405-433.

Lawson, A. (1988) On tests for spatial trend in a nonhomogeneous poisson process. J. Appl. Statist., 15, 225-234.

Lotwick, H. W. (1981) Spatial stochastic point processes. PhD Thesis. University of Bath, Bath.

Marr, D. (1982) Vision, p. vi. Englewood Cliffs: Prentice-Hall.

Miles, R. E. (1974) On the elimination of edge effects in planar sampling. In Stochastic Geometry (eds E. F. Harding and D. G. Kendall), pp. 228-247. Chichester: Wiley.

Ogata, Y. and Katsura, K. (1986) Point-process models with linearly parameterized intensity for application to earthquake data. J. Appl. Probab. A, 23, 291-310.

(1988) Likelihood analysis of spatial inhomogeneity for marked point patterns. Ann. Inst. Statist. Math., 40, 29-39.

Ripley, B. D. (1979) Tests of 'randomness' for spatial point patterns. J. R. Statist. Soc. B, 41, 368-374.

- (1981) Spatial Statistics. New York: Wiley.

(1988) Statistical Inference for Spatial Processes. Cambridge: Cambridge University Press.

Rosenfeld, A. and Pfalz, J. L. (1968) Distance functions on digital pictures. Pattn Recog., 1, 33-61.

Stoyan, D., Kendall, W. S. and Mecke, J. (1987) Stochastic Geometry and Its Applications. Chichester: Wiley.

Tufte, E. R. (1990) Envisioning Information, p. 17. Cheshire: Graphics Press.

Wilson, T. (ed.) (1990) Confocal Microscopy. London: Academic Press. 